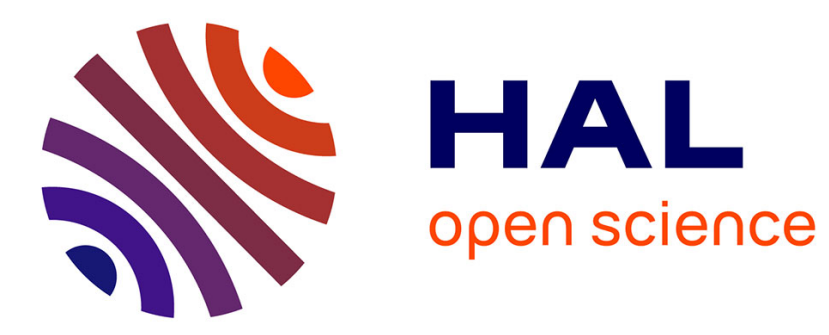

\title{
Non-interference in partial order models
}

\author{
Béatrice Bérard, Loïc Hélouët, John Mullins
}

\section{To cite this version:}

Béatrice Bérard, Loïc Hélouët, John Mullins. Non-interference in partial order models. ACM Transactions on Embedded Computing Systems (TECS), 2017, Application and Theory of Petri Nets and Other Models of Concurrency: Special Issue of Selected Papers from Petri Nets 2015, 16 (2), pp.44:144:34. 10.1145/2984639. hal-01379451

\section{HAL Id: hal-01379451 \\ https://hal.inria.fr/hal-01379451}

Submitted on 11 Oct 2016

HAL is a multi-disciplinary open access archive for the deposit and dissemination of scientific research documents, whether they are published or not. The documents may come from teaching and research institutions in France or abroad, or from public or private research centers.
L'archive ouverte pluridisciplinaire $\mathbf{H A L}$, est destinée au dépôt et à la diffusion de documents scientifiques de niveau recherche, publiés ou non, émanant des établissements d'enseignement et de recherche français ou étrangers, des laboratoires publics ou privés. 


\title{
Non-interference in partial order models
}

\author{
BÉATRICE BÉRARD, Sorbonne Universités, UPMC Univ. Paris 06, CNRS UMR 7606, LIP6 \\ LOÏC HÉLOUËT, INRIA Rennes \\ JOHN MULLINS, École Polytechnique de Montréal
}

\begin{abstract}
Non-interference (NI) is a property of systems stating that confidential actions should not cause effects observable by unauthorized users. Several variants of NI have been studied for many types of models, but rarely for true concurrency or unbounded models. This work investigates NI for High-level Message Sequence Charts (HMSC), a scenario language for the description of distributed systems, based on composition of partial orders. We first propose a general definition of security properties in terms of equivalence among observations of behaviors. Observations are naturally captured by partial order automata, a formalism that generalizes HMSCs and permits to assemble partial orders. We show that equivalence or inclusion properties for HMSCs (hence for partial order automata) are undecidable, which means in particular that NI is undecidable for HMSCs. We hence consider decidable subclasses of partial order automata and HMSCs. Finally, we define weaker local properties, describing situations where a system is attacked by a single agent, and show that local NI is decidable. We then refine local NI to a finer notion of causal NI that emphasizes causal dependencies between confidential actions and observations, and extend it to causal NI with (selective) declassification of confidential events. Checking whether a system satisfies local and causal NI and their declassified variants are PSPACE-complete problems.
\end{abstract}

CCS Concepts: $\bullet$ Software and its engineering $\rightarrow$ Software verification;

Additional Key Words and Phrases: Security, non-interference, partial orders, verification

ACM Reference Format:

Béatrice Bérard, Loïc Hélouët, and John Mullins, 2016. Non-interference in partial order models. ACM Trans. Embedd. Comput. Syst. V, N, Article A ( YYYY), 31 pages.

DOI: 0000001.0000001

\section{INTRODUCTION}

Context. Non-interference (NI) has been introduced to characterize the absence of harmful information flow in a system. It ensures that confidential actions of a system can not produce any effect visible by a public observer. The original notion of non-interference in [Goguen and Meseguer 1982] was expressed in terms of language equivalence for deterministic Mealy machines with confidential input and public output. Since then, several variants of information flow properties (IFP) have extended NI to non-deterministic models (transition systems, process algebra, Petri nets,...) and finer notions of observation (simple trace observation, deadlock or branching detection,....) to describe the various observational powers of an attacker. For a given system $S$, NI is usually defined as: $\pi_{V}(\llbracket S \backslash C \rrbracket) \approx \pi_{V}(\llbracket S \rrbracket)$ where $\approx$ denotes some behavioural system equivalence (language equivalence, bisimulation, ....), $\llbracket S \rrbracket$, the semantics of $S, \pi_{V}$, the projection on a subset $V$ of visible actions of the system, and $S \backslash C$, the model $S$ from which all confidential actions from $C$ are pruned. Intransitive non-interference (INI) relaxes NI to handle possible declassification of confidential ac-

This work is an extended version of [Bérard et al. 2015]. It was partially done while John Mullins was visiting the LIP6, Université Pierre \& Marie Curie. John Mullins is supported by the NSERC Discovery Individual grant No. 13321 (Government of Canada), the FQRNT Team grant No. 167440 (Quebec's Government) and the CFQCU France-Quebec Cooperative grant No. 167671 (Quebec's Government).

Author's addresses: B. Bérard, Sorbonne Universités, UPMC Univ. Paris 06, CNRS UMR 7606, LIP6, 75005, Paris, France; L. Hélouët, INRIA Rennes Campus de Beaulieu, 35041 Rennes Cedex, France; J. Mullins, Dept. of Computer \& Software Eng., École Polytechnique de Montréal, P.O. Box 6079, Station Centre-ville, Montreal (QC) Canada, H3C 3A7.

Permission to make digital or hard copies of all or part of this work for personal or classroom use is granted without fee provided that copies are not made or distributed for profit or commercial advantage and that copies bear this notice and the full citation on the first page. Copyrights for components of this work owned by others than ACM must be honored. Abstracting with credit is permitted. To copy otherwise, or republish, to post on servers or to redistribute to lists, requires prior specific permission and/or a fee. Request permissions from permissions@acm.org.

(c) YYYY ACM. 1539-9087/YYYY/-ARTA $\$ 15.00$

DOI : 0000001.0000001 
tions. It ensures that confidential actions of a system cannot produce any effect visible by a public observer unless they are declassified, causing so a harmless information flow. This issue has been addressed in [Rushby 1992], by comparing observations of visible actions in runs of a system (hence including runs containing non-declassified confidential actions), and observations of visible actions in runs of the same system that only contain confidential actions that are declassified afterwards. Most IFPs have been expressed as combinations of basic security predicates (BSPs) [Mantel 2000; 2001; D'Souza et al. 2011] or as a behavioral equivalence under observation contexts [Focardi and Gorrieri 2001]. A systematic presentation of IFPs can be found, e.g., in [Mantel 2000; 2001; Focardi and Gorrieri 2001].

Despite the fact that IFPs are always informally expressed in term of causality i.e., confidential activity should not cause observable effects on the public behavior, they are almost always formalized in terms of interleaving semantics [Busi and Gorrieri 2009; Gorrieri and Vernali 2011; Best et al. 2010; Best and Darondeau 2012] and hence, do not consider true concurrency or causality. This is clearly a lack in the formalization of IFPs for several reasons. First, from an algorithmic point of view, it is usually inefficient to compute a set of linearizations to address a problem that can be solved on an equivalent partial order representation. Second, a notion of interference based on equivalence that can distinguish between interleaved and concurrent sets of actions is more discriminating than a language based interference property. Last, from a practical point of view, an attacker of a system may gain more information if he knows that some confidential action has occurred recently in its causal past. Indeed, transactions in a distributed system can leave many traces (visited websites, cookies,...) on machines which are not a priori committed to protect confidential actions of third parties. To the best of our knowledge, [Baldan and Carraro 2014] is the first to address NI in a true concurrency setting: they characterized NI for Petri nets as a syntactic property of their unfoldings. However, the technique addresses only safe nets.

Very few results address IFPs for unbounded models. BSPs and NI are proved undecidable for pushdown systems, but decidability was obtained for small subclasses of context-free languages [D'Souza et al. 2011]. Decidability of a bisimulation-based strengthened version of NI called non-deducibility on composition (NDC) for unbounded Petri nets is proved in [Best et al. 2010]. A system satisfies NDC if observation of its visible actions remains indistinguishable from the observation of the system interacting with any environment. This result was extended in [Best and Darondeau 2012] to INI with selective declassification (INISD).

Contribution. This work considers IFPs for an unbounded true concurrency model, namely Highlevel Message Sequence Charts (HMSCs). This model, standardized by the ITU [ITU-T 2011], is well accepted to represent executions of distributed systems, where security problems are of primary concern. We first define a class of IFPs on HMSCs, as an inclusion relation on observations, following [Focardi and Gorrieri 2001; D'Souza et al. 2011] and [Bérard and Mullins 2014]. To keep IFPs within a true concurrency setting, observations of HMSCs are defined as partial orders. We define a new model called partial order automata (POA), that is powerful enough to recognize infinite sets of partial orders, and in particular observations of HMSCs. Unsurprisingly, most of IFPs and the simple NI property are undecidable for HMSCs. As a consequence, inclusion of partial order automata languages is undecidable. We then characterize decidable subclasses of the problem: inclusion of sets of orders generated by POA becomes decidable when the depicted behaviors do not allow observed processes to race each other. This is for instance the case when a POA describes an observation of visible events located on a single process. This also applies when the observed HMSC is locally synchronized meaning that within any iterated behavior, all processes synchronize at each iteration. We discuss the meaning of NI in a context where causal dependencies among event occurrences are considered. This leads to a new notion called causal interference for HMSCs. Causal interference detects interference as soon as an attacker can observe occurrences of confidential actions from visible events, and furthermore, one of the observed events causally depends on the confidential one. We finally relax causal interference in the context of declassification. We introduce intransitive causal non-interference that considers observable causal dependencies among 
confidential and visible events as safe, as soon as a declassification occurs in between. We show that all local variants of these problems are PSPACE-complete.

Outline. The basic models and definitions used in this paper are defined in Section 2. Observations, inclusion problems and non-interference are introduced in Section 3 for a single scenario and in Section 4 for HMSCs, where NI is proved undecidable. Section 4 introduces partial order automata as a way to recognize observations of HMSCs. We identify subclasses of HMSCs and POA where inclusion problems becomes decidable in Section 5. Then we consider local variants of interference problems in Section 6 and extend this framework to declassification in Section 7. We compare this work with some related approaches, and conclude in Section 8. Due to lack of space, several proofs are omitted or simply sketched, but can be found in an extended version at hal.inria.fr/hal-01280043.

\section{PRELIMINARIES}

In this section, we recall definitions of automata, partial orders and High-level Message Sequence Charts (HMSCs), with their associated languages.

Message Sequence Charts (MSCs) are formal representations of distributed executions, i.e., chronograms, that are frequently used to depict the behavior of a set of asynchronous communicating processes. This simple graphical representation emphasizes on messages and localization of actions, with partial order semantics. The model of HMSCs, standardized by the ITU [ITU-T 2011], was proposed to describe more elaborate behaviors of distributed systems, for instance those of communication protocols, by combining MSCs. HMSCs are used to describe sets of typical scenarios in distributed systems, and then serve as requirements. They can also be used as input to generate code skeletons for distributed systems. Hence, an information leak that appears in these early requirements is likely to be a feature of the final system. It is then interesting to find these leaks at early design stages. Another interesting point with HMSCs is their expressive power: they define behaviors of systems with asynchronous communications, which are not necessarily finite state systems and can not be captured by finite automata. They are also uncomparable with Petri nets. Answering interference questions for HMSCs provides security techniques for a whole class of infinite systems that can not be modeled with other formalisms.

\subsection{Finite automata and partial orders}

Let $\Sigma$ be a finite alphabet. A word over $\Sigma$ is a sequence $w=a_{1} a_{2} \ldots a_{n}$ of letters from $\Sigma$, and $\Sigma^{*}$ denotes the set of finite words over $\Sigma$, with $\varepsilon$ the empty word. A language is a subset $L$ of $\Sigma^{*}$. For a set $E$, we write $|E|$ for its cardinality. Given a relation $R \subseteq E \times E$ on $E$, we denote by $R^{*}$ the transitive and reflexive closure of $R$. A partial order on $E$ is a reflexive, transitive, and antisymmetric relation. Let $f_{1}$ and $f_{2}$ be two functions over disjoint domains $\operatorname{Dom}\left(f_{1}\right)$ and $\operatorname{Dom}\left(f_{2}\right)$. Then, $f_{1} \cup f_{2}$ denotes the function defined on $\operatorname{Dom}\left(f_{1}\right) \cup \operatorname{Dom}\left(f_{2}\right)$, that associates $f_{1}(x)$ with every $x \in \operatorname{Dom}\left(f_{1}\right)$ and $f_{2}(x)$ with every $x \in \operatorname{Dom}\left(f_{2}\right)$.

A Finite Automaton over alphabet $\Sigma$ is a tuple $\mathcal{A}=\left(S, \delta, s_{0}, F\right)$, where $S$ is a finite set of states, $s_{0} \in S$ is the initial state, $F \subseteq S$ is a set of accepting states, and $\delta \subseteq S \times \Sigma \times S$ is a transition relation. A word $w=a_{1} \ldots a_{n} \in \Sigma^{*}$, is accepted by $\mathcal{A}$ if there exists a sequence of transitions $\left(s_{0}, a_{1}, s_{1}\right)\left(s_{1}, a_{2}, s_{2}\right) \ldots\left(s_{n-1}, a_{n}, s_{n}\right)$ such that $s_{n} \in F$. It is well known that finite automata accept regular languages.

A Labeled Partial Order (LPO) over alphabet $\Sigma$ is a triple $O=(E, \leq, \alpha)$ where $(E, \leq)$ is a partially ordered set (poset) and $\alpha: E \rightarrow \Sigma$ is a labeling of $E$ by letters of $\Sigma$. The set of all LPOs over alphabet $\Sigma$ is denoted by $\operatorname{LPO}(\Sigma)$. For a subset of events $E^{\prime} \subseteq E$, the restriction of $O$ to $E^{\prime}$ is $O_{\mid E^{\prime}}=\left(E^{\prime}, \leq \cap\left(E^{\prime} \times E^{\prime}\right), \alpha_{\mid E^{\prime}}\right)$, where $\alpha_{\mid E^{\prime}}$ is the restriction of $\alpha$ to $E^{\prime}$. The set of predecessors of $E^{\prime}$ is $\downarrow\left(E^{\prime}\right)=\left\{f \in E \mid f \leq e\right.$ for some $\left.e \in E^{\prime}\right\}$ and the set of successors of $E^{\prime}$ is $\uparrow\left(E^{\prime}\right)=\left\{f \in E \mid e \leq f\right.$ for some $\left.e \in E^{\prime}\right\}$. The set $E^{\prime}$ is downward closed if $\downarrow\left(E^{\prime}\right)=E^{\prime}$, and upward closed if $\uparrow\left(E^{\prime}\right)=E^{\prime}$. A linear extension of an LPO $O=(E, \leq, \alpha)$ with $|E|=n$ is a sequence $r=e_{1} e_{2} \ldots e_{n}$ of all events of $E$ such that for every $j>k, e_{j} \not \leq e_{k}$. Linear extensions 
describe compatible sequences of events with the partial ordering. The size of LPO $O$, denoted by $|O|$, is $|E|$.

Let $O_{1}=\left(E_{1}, \leq_{1}, \alpha_{1}\right)$ and $O_{2}=\left(E_{2}, \leq_{2}, \alpha_{2}\right)$ be two LPOs over $\Sigma$. We write $O_{1} \sqsubseteq O_{2}$ if $O_{1}$ is a prefix of $O_{2}$ : there exists an injective mapping $h: E_{1} \rightarrow E_{2}$ such that $\alpha_{2}(h(e))=\alpha_{1}(e)$ for all $e \in E_{1}, h\left(E_{1}\right)$ is downward closed, and $e_{1} \leq_{1} f_{1}$ iff $h\left(e_{1}\right) \leq_{2} h\left(f_{1}\right)$. Moreover, $O_{1}$ is isomorphic to $O_{2}$, denoted by $O_{1} \equiv O_{2}$, if $O_{1} \sqsubseteq O_{2}$ and $O_{2} \sqsubseteq O_{1}$. A set of partial orders $Y$ contains another set of partial orders $X$, denoted by $\bar{X} \subseteq Y$, if for every $x \in X$, there exists $y \in Y$ such that $x \equiv y$. We will write $X \equiv Y$ if $X \subseteq Y$ and $Y \subseteq X$. We say that $X$ embeds into $Y$, denoted $X \sqsubseteq Y$ iff for every $x \in X$, there exists $y \in Y$ such that $x \sqsubseteq y$. Given an LPO $O=(E, \leq, \alpha)$, the covering of $O$ is a triple $(E, \prec, \alpha)$ where $\prec$ is the transitive and reflexive reduction of $\leq$, i.e., the smallest subset of $E \times E$ such that $\prec^{*}=\leq$. Since two orders are isomorphic iff their coverings are isomorphic, we often consider covering relations instead of orders in the rest of the paper. A causal chain in an LPO is a sequence of events $e_{1} e_{2} \ldots e_{k}$ such that $e_{i} \prec e_{i+1}$.
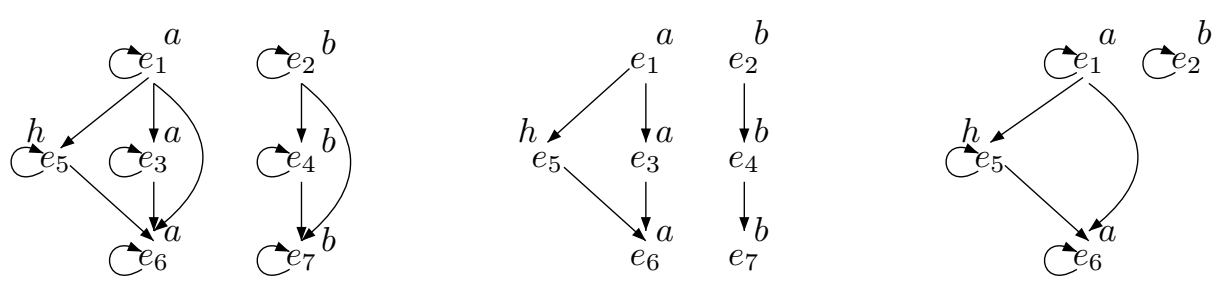

Fig. 1. An LPO (left) with its covering (middle) and a restriction (right)

Fig. 1 (left) shows an example of LPO, with set of events $E=\left\{e_{i} \mid 1 \leq i \leq 7\right\}$ and labels in $\{a, b, h\}$. A possible linear extension of this LPO is $e_{1} e_{3} e_{5} e_{2} e_{4} e_{7} e_{6}$, which corresponds to word aahbbba. Sequences of events $e_{1} e_{5} e_{6}, e_{1} e_{3} e_{6}$ and $e_{2} e_{4} e_{7}$ are causal chains of the LPO. Fig. 1 (middle) is a covering of this LPO, and Fig. 1 (right) is its restriction to $E^{\prime}=\left\{e_{1}, e_{2}, e_{5}, e_{6}\right\}$. As $E^{\prime}$ is upward closed, the order at the right of the figure is also a prefix of the leftmost order.

\subsection{High Level Message Sequence Charts}

Definition 2.1 (MSC). A Message Sequence Chart over finite sets $\mathbb{P}$ of processes, $\mathbb{M}$ of messages and finite alphabet $A$, is a tuple $M=\left(E,\left(\leq_{p}\right)_{p \in \mathbb{P}}, \alpha, \mu, \phi\right)$, where:

- $E$ is a finite set of events, partitioned as $E=E_{S} \uplus E_{R} \uplus E_{I}$, according to the type of event considered: message sending, reception, or internal atomic action that is, local events to a process which are not participating in communication;

- $\phi: E \rightarrow \mathbb{P}$ is a mapping associating with each event the process that executes it. Hence, the sets $E_{p}=\phi^{-1}(\{p\})$ for $p \in \mathbb{P}$, also form a partition of $E$;

- For each $p \in \mathbb{P}$, the relation $\leq_{p} \subseteq E_{p} \times E_{p}$ is a total ordering on events located on process $p$;

- $\mu \subseteq E_{S} \times E_{R}$ is a relation symbolizing message exchanges, such that if $(e, f) \in \mu$ with $e \in E_{p}$ and $f \in E_{q}$, then $p \neq q$. Furthermore, it induces a bijection from $E_{S}$ onto $E_{R}$, so with a slight abuse of notation, $(e, f) \in \mu$ is also written as $f=\mu(e)$. The relation $\leq_{M}=\left(\bigcup_{p \in \mathcal{P}} \leq_{p} \cup \mu\right)^{*}$ is a partial order on $E$;

- $\alpha$ is a mapping from $E$ to $\Sigma=(\mathbb{P} \times\{!, ?\} \times \mathbb{P} \times \mathbb{M}) \cup(\mathbb{P} \times A)$ and from $\mu$ to $\mathbb{M}$, associating a label with each event, and a message $\alpha(e, f)$ in $\mathbb{M}$ with each pair $(e, f) \in \mu$. The labeling is consistent with $\mu$ : if $f=\mu(e)$, with associated message $\alpha(e, f)=m$, sent by process $p$ to process $q$, then $\alpha(e)$ is written as $p ! q(m)$ and $\alpha(f)$ as $q ? p(m)$. If $e$ is an internal action $a$ located on process $p$, then $\alpha(e)$ is of the form $p(a)$. Summarising, $\Sigma$ may be written as $\{p ! q(m) \mid p, q \in \mathbb{P} \wedge m \in$ $\mathbb{M}\} \cup\{p ? q(m) \mid p, q \in \mathbb{P}, m \in \mathbb{M}\} \cup\{p(a) \mid p \in \mathbb{P}, a \in A\}$. The labeling is extended by morphism over $E^{*}$. 
As depicted in Fig. 2, we symbolize local events by bullets and communicating events as source and target of arrows labeled by a message name. The definition above implies that the triple $\left(E, \leq_{M}\right.$ $, \alpha)$ is an LPO over $\Sigma$, hence all notions related to posets also apply to MSCs. When clear from the context, we simply write $\leq$ instead of $\leq_{M}$. Given a subset $E^{\prime}$ of $E$, we denote by $M_{\mid E^{\prime}}$ the restriction of $M$ to $E^{\prime}$ (associated with the corresponding LPO restriction) and we denote by $M \backslash E^{\prime}$ the restriction of $M$ to $E \backslash E^{\prime}$. We denote by $\mathcal{M s c}(\mathbb{P}, \mathbb{M}, A)$ the set of all MSCs over the sets $\mathbb{P}$ of processes, $\mathbb{M}$ of messages, and alphabet $A$.

Definition 2.2. A linearization of MSC $M$ is a word $w \in \Sigma^{*}$ such that there exists a linear extension $r$ of $M$ with $w=\alpha(r)$. The language $\mathcal{L}(M)$ of $M$, is the set of linearizations of $M$.

The language of an MSC is hence a set of words over alphabet $\Sigma$. To design more elaborate behaviors, including choices and iterations, a key ingredient is sequential composition, that assembles MSCs processwise to form larger MSCs.

Definition 2.3. Let $M_{1}=\left(E_{1},\left(\leq_{1, p}\right)_{p \in \mathbb{P}}, \alpha_{1}, \mu_{1}, \phi_{1}\right)$ and $M_{2}=\left(E_{2},\left(\leq_{2, p}\right)_{p \in \mathbb{P}}, \alpha_{2}, \mu_{2}, \phi_{2}\right)$ be two MSCs defined over disjoint sets of events. The sequential composition of $M_{1}$ and $M_{2}$, denoted by $M_{1} \circ M_{2}$ is the MSC $M_{1} \circ M_{2}=\left(E_{1} \cup E_{2},\left(\leq_{1 \circ 2, p}\right)_{p \in \mathbb{P}}, \alpha_{1} \cup \alpha_{2}, \mu_{1} \cup \mu_{2}, \phi_{1} \cup \phi_{2}\right)$, where $\leq_{1 \circ 2, p}=\left(\leq_{1, p} \cup \leq_{2, p} \cup \phi_{1}^{-1}(\{p\}) \times \phi_{2}^{-1}(\{p\})\right)^{*}$.
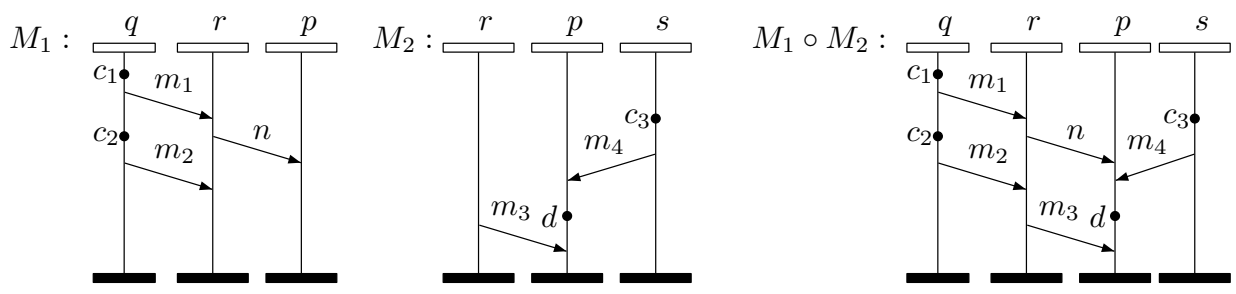

Fig. 2. An example of sequential composition of MSCs

In Fig. 2, MSCs $M_{1}$ and $M_{2}$ are assembled to produce MSC $M_{1} \circ M_{2}$. Intuitively, the relation $\leq_{1 \circ 2, p}$ in def. 2.3 expresses that all events in $M_{1}$ on process $p$ precede all events in $M_{2}$ on process $p$. This (associative) operation, also called concatenation, can be extended to $n$ MSCs. For a set $\mathcal{M}$ of MSCs, we denote by $\mathcal{M}^{\circ k}$ the set of all MSCs obtained by concatenation of $k$ MSCs in $\mathcal{M}$, with $\mathcal{M}^{\circ *}=\cup_{k>0} \mathcal{M}^{\circ k}$. Sequential composition is used to give a semantics to higher level constructs, namely HMSCs. Roughly speaking, an HMSC is a finite automaton where transitions are labeled by MSCs. It produces a set of MSCs obtained by concatenating MSCs that appear along paths.

Definition 2.4 (HMSC). A High-level MSC (HMSC) is a tuple $H=\left(N, \rightarrow, \mathcal{M}, n_{0}, F\right)$, where $N$ is a set of nodes, $\mathcal{M}$ is a finite set of MSCs, $\rightarrow \subseteq N \times \mathcal{M} \times N$ is a transition relation, $n_{0} \in N$ is the initial node, and $F$ is a set of accepting nodes.

As for any kind of automaton, paths and languages can be defined for HMSCs. A path of $H$ is a sequence $\rho=t_{1} t_{2} \ldots t_{k}$ such that for each $i \in\{1, \ldots, k\}, t_{i}=\left(n_{i}, M_{i}, n_{i}^{\prime}\right)$ is a transition in $\rightarrow$, with $n_{i}^{\prime}=n_{i+1}$ for each $i \leq k-1$. The path $\rho$ is a cycle if $n_{k}^{\prime}=n_{1}$. It is accepting if it starts from node $n_{0}\left(\right.$ i.e., $\left.n_{1}=n_{0}\right)$, and it terminates in a node of $F\left(n_{k}^{\prime} \in F\right)$.

Definition 2.5. Let $\rho=t_{1} t_{2} \ldots t_{k}$ be a path of an HMSC $H$. The MSC associated with $\rho$ is $M_{\rho}=h_{1}\left(M_{1}\right) \circ h_{2}\left(M_{2}\right) \cdots \circ h_{k}\left(M_{k}\right)$ where each $h_{i}$ is an isomorphism such that, if $M_{i}=\left(E_{i},\left(\leq_{i, p}\right)_{p \in \mathbb{P}}, \alpha_{i}, \mu_{i}, \phi_{i}\right)$, then $\forall j \neq i, h_{i}\left(E_{i}\right) \cap h_{j}\left(E_{j}\right)=\emptyset$.

More intuitively, the MSC associated with a path is obtained by concatenating MSCs encountered along this path after renaming the events to obtain disjoint sets of events. To simplify notation, we often drop the isomorphisms used to rename events, writing simply $M_{\rho}=M_{1} \circ M_{2} \circ \cdots \circ M_{k}$. 
With this automaton structure and the sequential composition of MSCs, an HMSC $H$ defines a set of accepting paths, denoted by $\mathcal{P}_{H}$, a set of MSCs $\mathcal{F}_{H}=\left\{M_{\rho} \mid \rho \in \mathcal{P}_{H}\right\}$, and a set of words obtained as the linearization language $\mathcal{L}(H)=\bigcup_{M \in \mathcal{F}_{H}} \mathcal{L}(M)$.

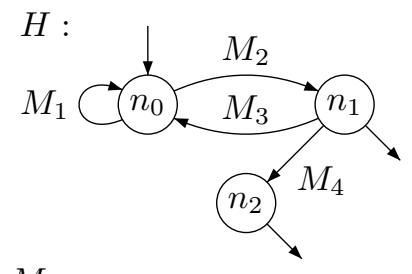

$M_{3}$ :

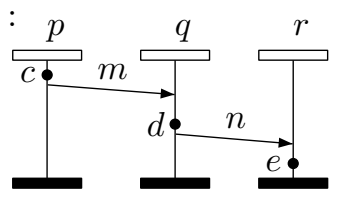

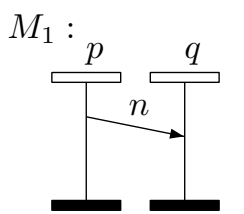

$M_{2}$ :
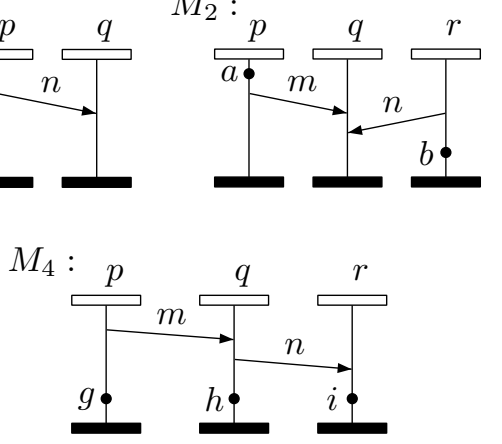

$M_{1} \circ M_{2} \circ M_{3} \circ M_{2} \circ M_{4}:$

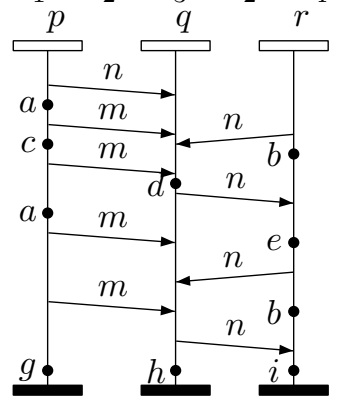

Fig. 3. An example of HMSC

Fig. 3 shows an example of HMSC, with transitions labeled by four MSCs $M_{1}, M_{2}, M_{3}, M_{4}$. The MSC $M_{1} \circ M_{2} \circ M_{3} \circ M_{2} \circ M_{4}$ shown at the right of the figure is an example of MSC generated by $H$.

It is well known that the linearization language of an HMSC is not necessarily regular, but rather a closure of a regular language under partial commutation, which yields many undecidability results (see for instance [Muscholl and Peled 1999; Caillaud et al. 2000]). This does not immediately mean that all IFPs are undecidable for HMSCs: Indeed, several classes of HMSCs with decidable properties have been identified and we later define non-trivial and meaningful subclasses of HMSCs and observations for which IFPs become decidable. In particular, the locally synchronized HMSCs defined below (and used to obtain decidability results in Section 5) have regular linearization languages [Alur and Yannakakis 1999]:

Definition 2.6. The communication graph of an $\operatorname{MSC} M=\left(E,\left(\leq_{p}\right)_{p \in \mathbb{P}}, \alpha, \mu, \phi\right)$ is the graph $(\mathbb{P}, \rightarrow)$ where $(p, q) \in \rightarrow$ if there exists a pair of events $(e, f) \in \mu$ such that $\phi(e)=p$ and $\phi(f)=q$. An HMSC $H$ is said locally synchronized iff for every cycle $\rho$ of $H$, the communication graph of $M_{\rho}$ is strongly connected.

Consider again the HMSC of Fig. 3. The communication graph of MSC $M_{2} \circ M_{3}$ is a graph with $\{p, q, r\}$ as vertices, and edges $\{(p, q),(r, q),(q, r)\}$. It is not strongly connected, so $H$ is not locally synchronized. Indeed, $H$ generates MSCs of the form $\left(M_{2} \circ M_{3}\right)^{\circ k} \circ M_{2}$. For any $k$, there is a linearization of $\mathcal{L}\left(\left(M_{2} \circ M_{3}\right)^{\circ k} \circ M_{2}\right)$ starting with $(a . p ! q(m) . c . p ! q(m))^{k}$. It reveals that in $H$, the process $p$ can arbitrarily repeat action sequences of the form $a . p ! q(m) . c . p ! q(m)$ without having to wait for any acknowledgment from other processes. This leads the represented system in a configuration where the remaining events of $\left(M_{2} \circ M_{3}\right)^{\circ k}$ on $q$ and $r$ have to be executed (and in particular receptions of sent messages). Hence, there is a race between $p$ and $q, r$ due to this cycle, and the linearization language of $H$ is not regular.

\section{OBSERVATION AND NON-INTERFERENCE FOR MSCs}

The power of an external observer can be described by an observation function, mapping every behavior of a system to some observables. In [Mantel 2000; 2001; D'Souza et al. 2011], observation functions are seen as specific language theoretic operations (projection, morphism, insertion, deletion of letters,...), and in [Bérard and Mullins 2014], they are combinations of rational operations (transductions, intersections, unions of languages).

In a distributed context, visible events can originate from different processes. In a distributed and asynchronous setting, the date at which an event is observed provides a linear ordering on observed events. However, this linear ordering does not necessarily correspond to an actual execution: two 
concurrent processes may execute events concurrently, or conversely, there might be some causal dependencies among observed events. This information on action dependencies might be available to observers: If the system is equipped with vector clocks (vectors maintained by each process to count the known number of events that other processes have executed, as proposed in [Mattern 1988]), one can also record causality in observations of a system. Hence, the natural and realistic notion of observation for distributed computations is a labeled partial order, where events that are not causally dependent are considered concurrent.

\subsection{Observations for MSCs}

Definition 3.1. An observation function is a mapping from $\mathcal{M s c}(\mathbb{P}, \mathbb{M}, A)$ to $L P O(B)$ for some alphabet $B$.

From this definition, any mapping from MSCs to LPOs can be called an observation. However, some observation functions are natural when considering IFPs. As proposed in [Mantel 2001] with the notion of views, the alphabet labeling events that occur during an execution of a system can be partitioned as $\Sigma=V \uplus C \uplus N$ with visible, confidential and internal (neutral) labels. Actions with labels in $V$ can be observed while actions labeled in $C$ are confidential and should be hidden. Internal actions have labels in $N$ and are not observable a priori, but need not be kept secret. Subsequently, depending on their labels, events are also called visible, confidential, or internal events.

Various observation functions can be defined from such a partition. The most natural ones are restrictions to visible events, and pruning of confidential actions, which are standard operations in language based non-interference literature, but need to be precisely defined in a partial order setting. Let $M=\left(E,\left(\leq_{p}\right)_{p \in \mathbb{P}}, \alpha, \mu, \phi\right)$ be an MSC with labeling alphabet $\Sigma$. We consider the following observation functions:

- identity: the identity $i d(M)=M$ outputs the same LPO as the executed MSC;

- Restriction: $\mathcal{O}^{V}(M)$ is the LPO obtained by restriction of $M$ to $\alpha^{-1}(V)$. Intuitively, $\mathcal{O}^{V}(M)$ represents the visible events and their causal dependencies that one may observe during the complete execution of $M$; Note that restriction to $\alpha^{-1}(V)$ suffices, as $\leq$ is transitive.

- Pruning: $\mathcal{O}_{\backslash C}^{V}(M)=\mathcal{O}^{V}\left(M \backslash \uparrow\left(\alpha^{-1}(C)\right)\right)$ is a function that prunes out the future of confidential events from $M$, leaving only the visible events and their causal dependencies, observed when no confidential event, nor their future, are executed within $M$;

- Localization: $\mathcal{O}^{p}(M)=\mathcal{O}^{V}\left(M_{\mid E_{p}}\right)$, for a given process $p \in \mathbb{P}$, is the observation of visible events of $M$ restricted to those events located on process $p$. Note that $\mathcal{O}^{p}(M)$ is a total order. In a distributed setting, $\mathcal{O}^{p}(M)$ is particularly interesting, as it represents the point of view of a single process $p \in \mathbb{P}$, considered as the attacker of the system. We hence assume no restriction on the set of events that can be executed and observed by $p$, and let $V=\Sigma_{p}=\alpha\left(E_{p}\right)$ when using $\mathcal{O}^{p}$.

\subsection{Non-interference for MSCs}

As noticed by [D'Souza et al. 2011] in a language setting, information flow properties of a system $S$ are usually defined as compositions of atomic propositions of the form $o p_{1}(S) \subseteq o p_{2}(S)$. Changing the observation functions $o p_{1}, o p_{2}$ (or the partition of $\Sigma$ ) leads to a variety of such atomic properties. Information flow properties of MSCs can be defined similarly.

Definition 3.2. Let $\mathcal{O}_{1}, \mathcal{O}_{2}$ be two observation functions over $\mathcal{M s c}(\mathbb{P}, \mathbb{M}, A)$. An MSC $M$ satisfies the inclusion property for $\mathcal{O}_{1}, \mathcal{O}_{2}$, written $\sqsubseteq \mathcal{O}_{1}, \mathcal{O}_{2}(M)$, if $\mathcal{O}_{1}(M) \sqsubseteq \mathcal{O}_{2}(M)$.

Very often, interference is informally described as causal dependencies between confidential actions and observable ones, but formalized in terms of languages comparison, i.e., with interleaved representations that miss information on concurrency and causality. Consider for instance the basic HMSC of Fig. 4. It generates only two MSCs: $M_{\text {high }}$ and $M_{\text {low }}$. Now assume that an attacker of the system can only observe actions $a$ and $b$, that action $h$ is a confidential action, and that all other events are unobservable. In an interleaving setting, the attacker may observe words $a b$ and $b a$, no 


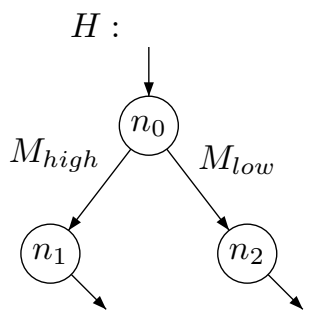

$M_{\text {high }}$ :

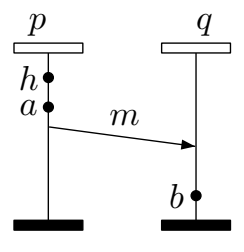

$M_{\text {low }}:$

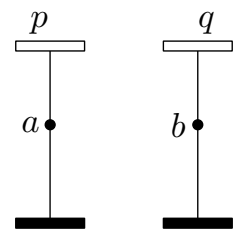

Fig. 4. A HMSC showing the discriminating power of partial order representation.

matter whether $h$ occurs or not hence, the system does not leak information. Now, if an attacker has the capability to observe $a, b$ and their causal dependencies, then observing that $a$ precedes $b$ reveals the occurrence of $h$. So, in a setting where causal dependencies can be observed, the system leaks information. For a single MSC $M$, the notion of non-interference is defined as a comparison of partial orders:

Definition 3.3. An MSC $M$ over $\Sigma=C \uplus V \uplus N$ is non-interferent if $\mathcal{O}^{V}(M) \equiv \mathcal{O}_{\backslash C}^{V}(M)$. Otherwise $M$ is said interferent.

We now show that interference in a single MSC can be characterized in terms of causal dependencies from confidential events in $C$ to visible ones in $V$. We then show in Section 3.3 that checking existence of such dependencies can be performed via coloring of events. For a single MSC, comparing observations $\mathcal{O}^{V}$ and $\mathcal{O}_{Y C}^{V}$ as defined above highlights dependencies between confidential and visible actions. Hence, interference in a single MSC can be defined through causality:

PROPOSITION 3.4. Let $M$ be an MSC over $\Sigma=C \uplus V \uplus N$ and set of events $E$. Then, $M$ is interferent if and only if there exists two events $e, f$ such that $\alpha(e) \in C, \alpha(f) \in V$, and $e \leq f$.

This result will be used to define interference in terms of MSC coloring, and also to prove that this coloring is compositional.

\subsection{Interference detection by coloring}

The relation between causal dependencies and interference calls for a graphical interpretation of interference in MSCs, represented as a propagation of a black token inherited from confidential actions along causal dependencies. Intuitively, any confidential action and successors of actions marked with a black token are also marked with a black token and every process containing a black action is also marked as black. This black and white coloring representation of MSCs will be conveniently used later to detect information flows in HMSCs.

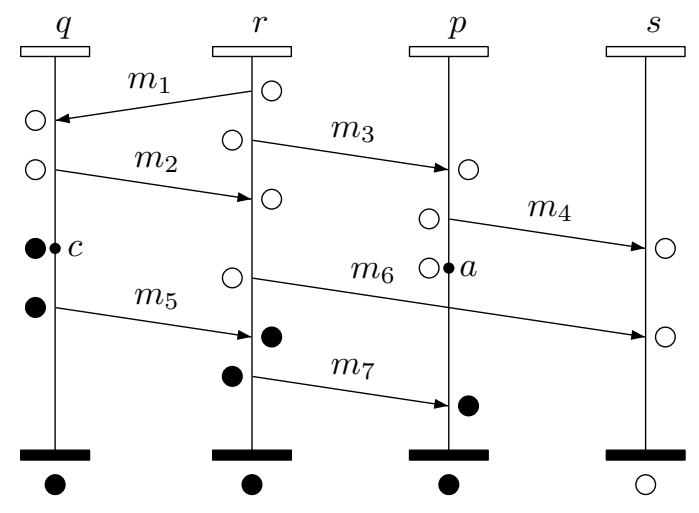

Fig. 5. An MSC $M_{b w}$ tagged with black and white tokens 
Definition 3.5 (MSC and process coloring). Let $M$ be an MSC over an alphabet $\Sigma=C \uplus V \uplus N$ and a set of events $E$. An event $e \in E$ is black if $\alpha(\downarrow(e)) \cap C \neq \emptyset$, and white otherwise. A process $p \in \mathbb{P}$ is black after $M$ (resp. white after $M$ ) if there exists a black event located on $p$ (resp. no black event on $p$ ).

Fig. 5 shows a coloring of an MSC $M_{b w}$ in black and white. The alphabet of confidential actions is $C=\{q(c)\}$, i.e. it contains an atomic action $c$ executed by process $q$. We attach a black token to every black event and a white token to other events. Similarly, we indicate with a black/white token below process lines whether a process has met a black token during its execution. Intuitively, a black process can detect occurrences of confidential events, as it executes events that are causal consequences of confidential events. In this example, process $p$ can detect occurrences of $c$ (it is black after $M_{b w}$ ), but process $s$ cannot.

In this coloring setting, interference has an obvious operational meaning: an MSC is interferent iff it contains a visible black event. The MSC $M_{b w}$ depicted in Fig. 5, is interferent as $p ? r\left(m_{7}\right) \in V$. Hence, deciding if an MSC is interferent reduces to searching a path from a confidential event to a visible one in an acyclic graph where events are seen as vertices and pairs of events $(e, f)$ in $\left(\cup_{p \in \mathcal{P}} \leq_{p}\right) \cup \mu$ as edges. Since an event has at most two immediate successors, the graph to consider has at most $n=\left|E_{M}\right|$ vertices and $2 \cdot n$ edges. Hence, interference detection in a MSC can be performed in linear time as a graph exploration starting from confidential events. Another interesting property is that deciding the black/white status of a process after a sequence of MSCs of arbitrary size can be performed in bounded memory.

Proposition 3.6. Let $M_{1}, M_{2}$ be two MSCs with labels in $\Sigma=C \uplus V \uplus N$. Then, process $p \in \mathbb{P}$ is black after $M_{1} \circ M_{2}$ iff it is black after $M_{1}$, or it is black after $M_{2}$, or there exists a process $q$ black after $M_{1}$ and a pair of events $e \leq f$ in $M_{2}$ such that $e$ is located on $q$ and $f$ is located on $p$.

This important property means that it is sufficient to remember the black/white status of each process after concatenation $M_{1} \circ \cdots \circ M_{k}$ along a path of an HMSC to compute the status of process $p$ after concatenation $M_{1} \circ \cdots \circ M_{k} \circ M_{k+1}$.

\section{OBSERVATIONS ON HMSCS AS PARTIAL ORDER AUTOMATA}

In this section, we first discuss extending observation functions from MSCs to HMSCs and show that the inclusion problem as well as non-interference are undecidable for HMSCs. We also remark that some observation functions on HMSCs can be obtained by assembling partial orders obtained by observation of MSCs encountered along a path, but with composition operators that are more powerful than sequential composition of MSCs. This suggests the definition of Partial Order Automata (POA) that are finite automata where transitions are labeled by LPOs. To increase the expressive power of this model, we introduce various ways of assembling the partial orders appearing along paths through composition operators and selection functions. The main purpose of this section is to present the material needed in Section 5 where we prove that non-interference is decidable for the subclass of locally synchronized HMSCs. This result is obtained by (1) building two partial order automata $\mathcal{A}_{H, \mathcal{O}_{1}}$ and $\mathcal{A}_{H, \mathcal{O}_{2}}$ associated with a locally synchronized HMSC $H$, respectively accepting observations $\mathcal{O}_{1}(H)$ and $\mathcal{O}_{2}(H)$ and (2) proving that in this case, the inclusion problem $\mathcal{O}_{1}(H) \subseteq \mathcal{O}_{2}(H)$ is decidable. In this section, we mainly identify sufficient conditions on the observation functions to achieve point (1) above, while decidability is proved in the next section.

\subsection{Extending observations to HMSCs}

In order to extend an observation $\mathcal{O}$ to an HMSC $H$, a first way consists in applying $\mathcal{O}$ to all MSCs in $\mathcal{F}_{H}$, defining $\mathcal{O}(H)=\left\{\mathcal{O}(M) \mid M \in \mathcal{F}_{H}\right\}$. In particular : $\mathcal{O}^{V, \circ}(H)=\left\{\mathcal{O}^{V}(M) \mid M \in\right.$ $\left.\mathcal{F}_{H}\right\}, \mathcal{O}_{\backslash C}^{V, \circ}(H)=\left\{\mathcal{O}_{\backslash C}^{V}(M) \mid M \in \mathcal{F}_{H}\right\}$, and $\mathcal{O}^{p, \circ}(H)=\left\{\mathcal{O}^{p}(M) \mid M \in \mathcal{F}_{H}\right\}$. Extending Definitions 3.2 and 3.3 to HMSCs, we have: 
Definition 4.1. An HMSC $H$ satisfies the inclusion problem for $\mathcal{O}_{1}, \mathcal{O}_{2}$ (written $\sqsubseteq \mathcal{O}_{1}, \mathcal{O}_{2}(H)$ ) if $\mathcal{O}_{1}(H) \subseteq \mathcal{O}_{2}(H)$. It is non-interferent if $\mathcal{O}_{\backslash C}^{V, \circ}(H) \equiv \mathcal{O}^{V, \circ}(H)$.

The example of Fig. 4 is a typical situation where $\mathcal{O}_{\backslash C}^{V, \circ}(H)$ and $\mathcal{O}^{V, \circ}(H)$ differ: $\mathcal{O}_{\backslash C}^{V, o}(H)$ contains a single LPO with two concurrent events labeled $a$ and $b$, while $\mathcal{O}^{V, o}(H)$ contains in addition a second LPO with two events labeled $a$ and $b$ such that $a<b$. Unfortunately, the observation functions above do not take into account the structure of the HMSC generating $\mathcal{F}_{H}$, and furthermore, they are not necessarily compositional. In general, an observation function $\mathcal{O}$ is not a morphism with respect to the concatenation, that is, $\mathcal{O}\left(M_{1} \circ M_{2}\right) \neq \mathcal{O}\left(M_{1}\right) \circ \mathcal{O}\left(M_{2}\right)$. This drawback was already observed in [Genest et al. 2003] for projections of MSCs: in general, $\mathcal{O}^{V}\left(M_{1} \circ M_{2}\right) \neq \mathcal{O}^{V}\left(M_{1}\right) \circ \mathcal{O}^{V}\left(M_{2}\right)$. Consider for instance the example of Fig. 2 with $V=\left\{c_{1}, c_{2}, c_{3}, d\right\}$. One can easily see that $\mathcal{O}^{V}\left(M_{1} \circ M_{2}\right)$ is an LPO in which the event carrying label $c_{1}$ precedes the event carrying label $b$. This causal dependency does not exist in $\mathcal{O}^{V}\left(M_{1}\right) \circ \mathcal{O}^{V}\left(M_{2}\right)$. Hence, checking inclusion for HMSCs may require to consider properties of complete sequences of MSCs as a whole, raising algorithmic difficulties, or even undecidability. Other ways to extend observations to HMSCs, are to assemble observations of MSCs piecewise, following the automaton structure of HMSCs, or to forbid MSCs containing confidential events:

$$
\begin{aligned}
& \mathcal{O}^{V, \bullet}(H)=\left\{\mathcal{O}^{V}\left(M_{1}\right) \circ \cdots \circ \mathcal{O}^{V}\left(M_{k}\right) \mid M_{1} \circ \cdots \circ M_{k} \in \mathcal{F}_{H}\right\}, \\
& \mathcal{O}_{i C}^{V, \bullet}(H)=\left\{\mathcal{O}^{V}\left(M_{1} \circ \cdots \circ M_{k}\right) \mid M_{1} \circ \cdots \circ M_{k} \in \mathcal{F}_{H} \wedge \forall i, \alpha\left(E_{i}\right) \cap C=\emptyset\right\}, \\
& \mathcal{O}^{p, \bullet}(H)=\left\{\mathcal{O}^{p}\left(M_{1}\right) \cdots \cdots \mathcal{O}^{p}\left(M_{k}\right) \mid M_{1} \circ \cdots \circ M_{k} \in \mathcal{F}_{H}\right\},
\end{aligned}
$$

where concatenation of LPOs is performed processwise like for MSCs. The observation $\mathcal{O}_{C}^{V, \bullet}(H)$ is of particular interest, as it describes observations of MSCs in $\mathcal{F}_{H}$ that do not contain MSCs with confidential events. Also note that, since $\mathcal{O}^{p}(M)$ is a total order, $\mathcal{O}^{p}$ satisfies the morphism property, which implies $\mathcal{O}^{p, \circ}(H)=\mathcal{O}^{p, \bullet}(H)$.

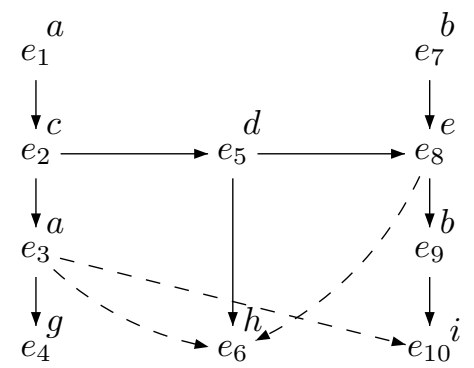

Fig. 6. Projection of MSC $M_{1} \circ M_{2} \circ M_{3} \circ M_{2} \circ M_{4}$ on $V=\{a, b, c, d, e, f, g, h, i\}$

The difference between observation functions is illustrated on the MSC obtained from $H$ in Fig. 3 (right), with $V=\{a, b, c, d, e, f, g, h, i\}$ as alphabet of visible events. For $M=M_{1} \circ M_{2} \circ M_{3} \circ$ $M_{2} \circ M_{4}$, the (covering of) observation $\mathcal{O}^{V}(M) \in \mathcal{O}^{V, \circ}(H)$ is depicted in Fig. 6. The observation covering for the same path in $\mathcal{O}^{V, \bullet}(H)$ is obtained from this diagram by removing the dashed dependencies from $e_{8}$ to $e_{6}$ and from $e_{3}$ to $e_{10}$ and to $e_{6}$. This example clearly shows that concatenation of projections of MSCs and projections of concatenations of MSCs differ. In our example, this mainly comes from the fact that dependencies stemming from message $m$ in $M_{2}$ and messages $m, n$ in $M_{4}$ are lost during projection. Observation $\mathcal{O}^{p, \bullet}(M)$ is simply the restriction of this order to $e_{7}, e_{8}, e_{9}, e_{10}$.

Even when a projection of an HMSC is an HMSC language (i.e., a language recognizable by an HMSC), equivalence, inclusion or emptiness of intersection are undecidable. HMSC languages are not always regular and the observation of an HMSC needs not be regular either. In fact, due to the 
close relationship between HMSCs and Mazurkiewicz traces, most properties requiring to compare languages or partial order families are undecidable for HMSCs ([Caillaud et al. 2000; Muscholl and Peled 1999; 2000]). So, given two HMSCs $H_{1}$ and $H_{2}$, one can not decide if $\mathcal{L}\left(H_{1}\right) \subseteq \mathcal{L}\left(H_{2}\right)$, nor if $\mathcal{F}_{H_{1}} \subseteq \mathcal{F}_{H_{2}}$. This yields the following result:

THEOREM 4.2. The inclusion problem $\sqsubseteq \mathcal{O}_{1}, \mathcal{O}_{2}(H)$ is undecidable for $H$ an $H M S C$ and $\mathcal{O}_{1}, \mathcal{O}_{2}$ two observation functions.

PROOF. The proof is a reduction from the inclusion problem for partial order families generated by HMSCs. For two HMSCs $H_{1}$ and $H_{2}$, the question of whether $\mathcal{F}_{H_{1}} \subseteq \mathcal{F}_{H_{2}}$ is undecidable [Caillaud et al. 2000].

Let $H_{1}=\left(N_{1}, \rightarrow_{1}, \mathcal{M}_{1}, n_{0,1}, F_{1}\right)$ and $H_{2}=\left(N_{2}, \rightarrow_{2}, \mathcal{M}_{2}, n_{0,2}, F_{2}\right)$ be two HMSCs, defined over an alphabet of visible actions $V$, and with a set $\mathbb{P}$ containing at least two processes. We build an HMSC $H$, that behaves like $H_{1}$ or $H_{2}$ if a confidential action can occur, and like $H_{2}$ otherwise, and choose observation functions $\mathcal{O}_{1}=\mathcal{O}^{V, \circ}, \mathcal{O}_{2}=\mathcal{O}_{\backslash C}^{V \text {, }}$. Then inclusion $\sqsubseteq \mathcal{O}_{1}, \mathcal{O}_{2}(H)$ holds iff $\mathcal{F}_{H_{1}} \subseteq \mathcal{F}_{H_{2}}$.

Let $c$ be a new confidential action and $P_{c} \notin \mathbb{P}$ a new process. We define $M_{c}$ as the MSC containing the single atomic action $c$ on process $P_{c}$, as illustrated on Fig. 7 (middle). The new HMSC $H=$ $\left(N_{1} \uplus N_{2}, \rightarrow, \mathcal{M}, n_{0,2}, F_{1} \uplus F_{2}\right)$ is defined over alphabet $\Sigma^{\prime}=V \cup C$, where $C=\left\{P_{c}(c)\right\}$, as follows: $\mathcal{M}=\mathcal{M}_{1} \uplus \mathcal{M}_{2} \uplus\left\{M_{c}\right\}$ and $\rightarrow=\rightarrow_{1} \uplus \rightarrow_{2} \uplus\left\{\left(n_{0,2}, M_{c}, n_{0,1}\right)\right\}$, as illustrated on the left part of Fig. 7. For $\mathcal{O}_{1}=\mathcal{O}^{V, \circ}$ and $\mathcal{O}_{2}=\mathcal{O}_{\backslash C}^{V, \circ}$, we have $\mathcal{O}_{2}(H)=\mathcal{F}_{H_{2}}$ and $\mathcal{O}_{1}(H)=\mathcal{F}_{H_{1}} \cup \mathcal{F}_{H_{2}}$. Thus $\sqsubseteq \mathcal{O}_{1}, \mathcal{O}_{2}(H)$ if and only if $\mathcal{F}_{H_{1}} \subseteq \mathcal{F}_{H_{2}}$, which concludes the proof.

Note that undecidability of inclusion problems is not due to a particular choice of observation function: a similar proof is obtained for $\mathcal{O}_{1}=\mathcal{O}^{V, \circ}$ or $\mathcal{O}_{1}=\mathcal{O}^{V, \bullet}$ and $\mathcal{O}_{2}=\mathcal{O}_{\backslash C}^{V \text {, }}$, by replacing $M_{c}$ by an MSC $M_{c}^{\prime}$ in which process $P_{c}$ sends a message to all other processes after performing action $c$, as depicted on the right of Fig. 7 .
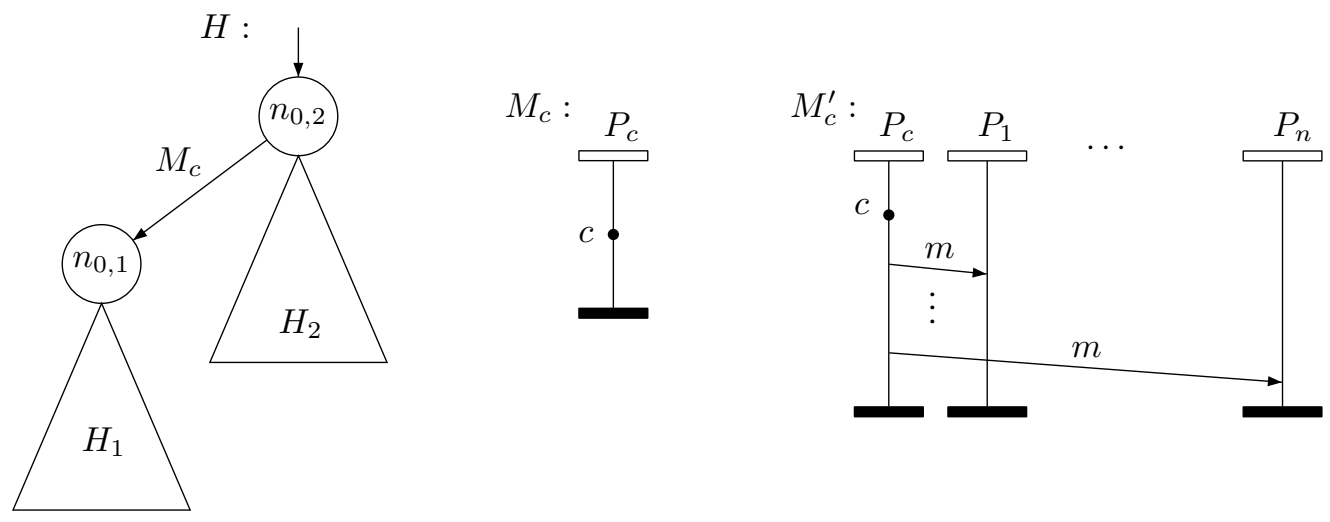

Fig. 7. Non-interference in HMSCs as an inclusion problem

This result extends to non-interference properties:

COROLlary 4.3. Non-interference for HMSCs is undecidable.

PROOF. Consider again the example HMSC $H$ built in the proof of theorem 4.2 (and shown in Fig. 7). Recall that observable events are those in $H_{1}$ and $H_{2}$, while the only confidential event in $H$ is the one labeled by $c$. The chosen observation functions are $\mathcal{O}^{V, \circ}$ and $\mathcal{O}_{\backslash C}^{V \text {, }}$. If an algorithm answers the interference question for any HMSC, then it can be used to check isomorphism of $\mathcal{F}_{H_{1}}$ 
and $\mathcal{F}_{H_{2}}$ for any pair of HMSC $H_{1}, H_{2}$ (by building the HMSC $H$ as in the proof of theorem 4.2). Thus, the interference problem for HMSCs is undecidable.

\subsection{Partial order automata}

While HMSCs assemble finite MSCs to produce larger MSCs, i.e. particular LPOs, inclusion and interference properties do not compare MSCs but observations of MSCs. As mentioned above, projections of HMSCs are not in general HMSCs [Genest et al. 2003], hence observations of HMSCs are not HMSCs either. To compare the orderings (or their coverings) obtained by observation of a set of MSCs, we need more general structures. We propose in this section a model called Partial Order Automata (POA), that assemble partial orders (or their coverings). Partial order automata are automata labeled by finite orders, and at each transition, the way to assemble the labeling order depends on a glueing operator attached to this transition, and on a set of memorized events. This model is more general than HMSCs, where the glueing operator is the same (sequential composition $\circ$ ) for every transition. However, in this paper, we will not use the whole expressive power of partial order automata, and we will only use POA to define projections of partial order families generated by HMSCs. Yet, we need a model that is more expressive than HMSCs: as explained earlier, the observation of a sequential composition of MSC differs from the sequential composition of their projections.

Definition 4.4 (Composition operator). A composition operator for partial orders is an operator $\otimes: L P O(\Sigma) \times 2^{\mathbb{E}} \times L P O(\Sigma) \rightarrow L P O(\Sigma)$, where $\mathbb{E}$ is a set of events, that computes a partial order from a pair of partial orders $O_{1}, O_{2}$ and a subset of identified events $\mathrm{Mem}_{1}$ from $O_{1}$. The result is denoted by $\left(O_{1}, M e m_{1}\right) \otimes O_{2}$. In practice, the operation is performed from coverings of $O_{1}$ and $O_{2}$ and produces a covering of the result.

A selection function is a function $\Pi$ associating with a partial order $O=(E, \leq, \alpha)$ a subset of events $E^{\prime} \subseteq E$. A selection function $\Pi$ is monotonic if, for every pair of orders $O_{1} \sqsubseteq O_{2}$, with $O_{1}=\left(E_{1}, \leq_{1}, \alpha_{1}\right)$ and $O_{2}=\left(h\left(E_{1}\right) \uplus E_{2}, \leq_{2}, \alpha_{2}\right)$, where $h$ is the injective mapping between the sets of events proving $O_{1} \sqsubseteq O_{2}$, then $\Pi\left(O_{2}\right) \subseteq h\left(\Pi\left(O_{1}\right)\right) \uplus E_{2}$. Function $\Pi$ is a finite memory function if there exists $K \in \mathbb{N}$ such that $|\Pi(O)| \leq K$ for every LPO $O \in L P O(\Sigma)$.

Selection functions are used to memorize events of interest during the construction of a covering relation by a partial order automaton. Intuitively, given two coverings $O_{1}=\left(E_{1}, \prec_{1}, \alpha_{1}\right)$ and $O_{2}=$ $\left(E_{2}, \prec_{2}, \alpha_{2}\right)$, and a memorized subset of events $\mathrm{Mem}_{1}$ in $E_{1}$ then $O=\left(O_{1}, \mathrm{Mem}_{1}\right) \otimes \mathrm{O}_{2}$ is a covering $O=(E, \prec, \alpha)$ where $E=E_{1} \uplus E_{2}, \alpha=\alpha_{1} \cup \alpha_{2}$, and $\prec$ is a covering relation that contains $\prec_{1} \cup \prec_{2}$ and such that $\prec \backslash\left(\prec_{1} \cup \prec_{2}\right) \subseteq$ Mem $_{1} \times E_{2}$ (the operator only glues events from the selection and events from the newly added order). Let us consider a monotonic selection function $\Pi$, and a sequence of composition operations. Slightly abusing our notation, we write $O_{1} \otimes_{1} O_{2}$ instead of $\left(O_{1}, \Pi\left(O_{1}\right)\right) \otimes_{1} O_{2}$, and similarly for sequences of compositions, we write $O=O_{1} \otimes_{1} O_{2} \otimes_{2} \cdots \otimes_{k-1} O_{k}$, and leave the selection process implicit. For monotonic selection functions, remembering previously memorized events suffices to compute a new memory. We can hence safely write $\Pi(M e m \otimes O)$ to define the set of events memorized after concatenation of $O$ to any order $O^{\prime}$ such that $\Pi\left(O^{\prime}\right)=$ Mem.

In the rest of the paper, we consider composition operators that assemble multiple copies from a finite set of orders, i.e., compositions of the form $O=O_{1} \otimes_{1} O_{2} \otimes_{2} \cdots \otimes_{k-1} O_{k}$ where each $O_{i}$ is a copy from a finite set of LPOs $\mathbb{L}$, and $\otimes_{1}, \cdots \otimes_{k-1}$ are composition operators. To distinguish multiple copies of an order and of its events, we denote by $L^{(j)}$ the $j^{\text {th }}$ occurrence of order $L=$ $\left(E_{L}, \leq_{L}, \alpha_{L}\right) \in \mathbb{L}$, and by $e^{(j)}$ the $j^{t h}$ occurrence of some event $e \in E_{L}$.

Example 4.5. An example of selection function is the function, denoted by MaxEvt, that selects the last occurrence of each event of each order in $\mathbb{L}$. For a sequence $O=O_{1} \otimes_{1} O_{2} \otimes_{2} \cdots \otimes_{k-1} O_{k}$ and $L \in \mathbb{L}$, then $\operatorname{MaxEvt}(O)=\cup_{L \in \mathbb{L}}\left\{\left.e^{(j)}\left|e \in E_{L} \wedge\right| O\right|_{L}=j\right\}$, where $|O|_{L}$ is the number of occurrences of $L$ in $O$. One can notice that MaxEvt is monotonic, and returns a finite set of events regardless of the size of the considered sequence of compositions. 

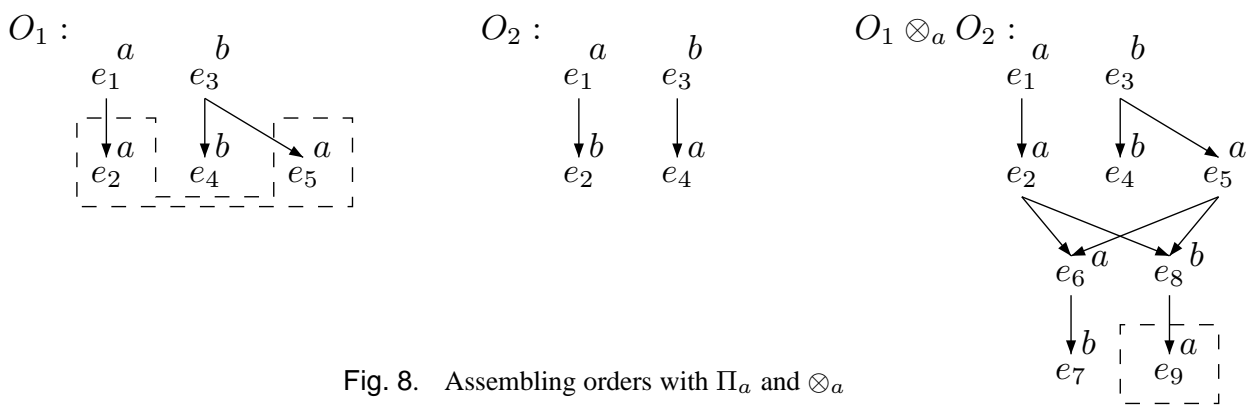

Fig. 8. Assembling orders with $\Pi_{a}$ and $\otimes_{a}$

Example 4.6. Consider the example of Fig. 8. Let us define a selection function $\Pi_{a}$ that remembers the maximal occurrences of events carrying label $a$ that are maximal with respect to the ordering, and a composition operator $\otimes_{a}$ that merges an order by creating dependencies from all memorized events to minimal events of the appended order. The figure shows two orders $O_{1}, O_{2}$, the set of events kept in memory (in dashed parts) and the assembled order $O_{1} \otimes_{a} O_{2}$.

Definition 4.7. A Partial Order Automaton (POA) over a finite set OPS of composition operators is a tuple $\mathcal{A}=\left(Q, \longrightarrow, \mathbb{L}, q_{0}, F, \Lambda, \Pi\right)$ where $Q$ is a finite set of states, $q_{0} \in Q$ is the initial state, $F \subseteq Q$ is a set of final states, $\mathbb{L}$ is a finite set of LPOs, $\longrightarrow \subseteq Q \times \mathbb{L} \times Q$ is a set of transitions, $\Lambda$ is a mapping associating with each transition an operator from $O P S$, and $\Pi$ is a monotonic selection function. The transition relation is deterministic: for each $L \in \mathbb{L}$, and each $q \in Q$, there is at most one $q^{\prime} \in Q$ such that $\left(q, L, q^{\prime}\right) \in \longrightarrow$.

For every path $\rho=q_{0} \stackrel{O_{1}}{\longrightarrow} q_{1} \stackrel{O_{2}}{\longrightarrow} q_{2} \ldots q_{k-1} \stackrel{O_{k}}{\longrightarrow} q_{k}$ of $\mathcal{A}$, one can compute an LPO $O_{\rho}$ assembled as $O_{\rho}=O_{1} \Lambda\left(q_{1}, O_{2}, q_{2}\right) O_{2} \ldots \Lambda\left(q_{k-1}, O_{k}, q_{k}\right) O_{k}$. For readability, we often omit the specific operators used to assemble orders, and simply write $O_{\rho}=O_{1} \otimes O_{2} \cdots \otimes O_{k}$. For two events $e$ and $f$, we write $e \leq_{\rho} f$ when $e$ precedes $f$ in the partial order $O_{\rho}$. The partial order language of a POA $\mathcal{A}$ is the set of orders obtained by assembling orders along accepting paths of $\mathcal{A}$, and is denoted by $\mathcal{F}_{\mathcal{A}}$. The linearization language of $\mathcal{A}$ is the set of linearizations of orders in $\mathcal{F}_{\mathcal{A}}$.
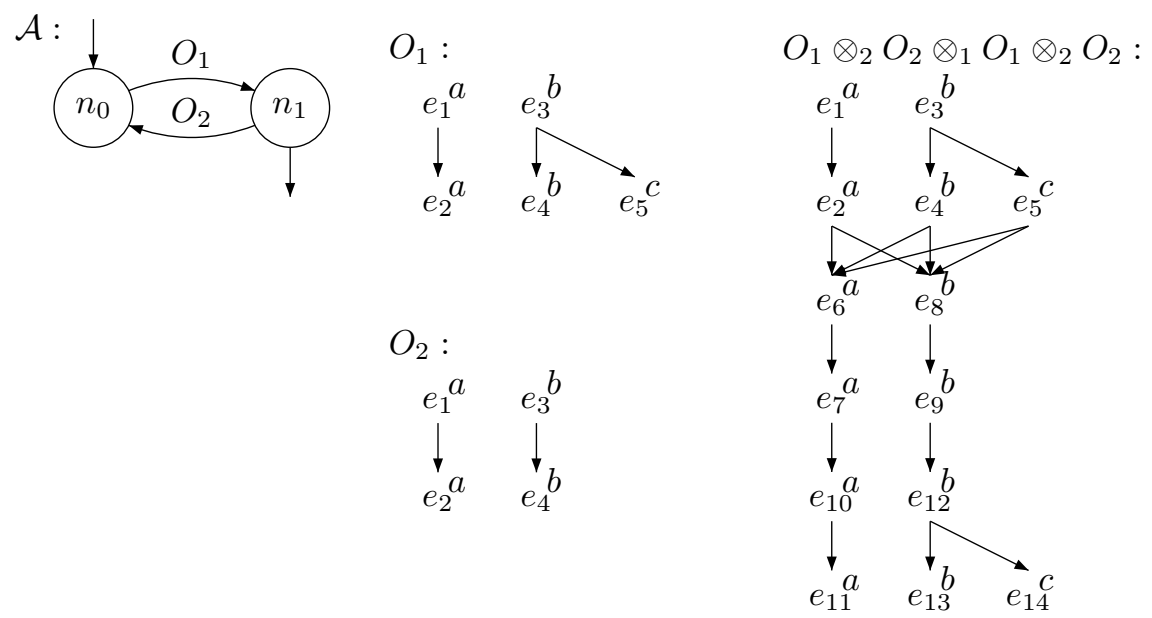

Fig. 9. An example of Partial Order Automaton

Consider the POA $\mathcal{A}$ depicted in Fig. 9, over the set of orders $\mathbb{L}=\left\{O_{1}, O_{2}\right\}$. We choose a selection function $\Pi$ that remembers all maximal events in the order generated so far, and we let $\otimes_{1}=\Lambda\left(n_{0}, O_{1}, n_{1}\right)$ be the operator that glues minimal events of the appended order to the maximal events formerly memorized, and $\otimes_{2}=\Lambda\left(n_{1}, O_{2}, n_{0}\right)$ be the operator that creates a precedence 
relation from every event memorized with label $\alpha$ to the minimal events with label $\alpha$ in the appended order. With these operators, our POA generates for instance the order $O_{1} \otimes_{2} O_{2} \otimes_{1} O_{1} \otimes_{2} O_{2}$ shown at the right of the figure.

First note that deterministic finite automata are particular cases of POA where each order labeling a transition is reduced to a single event, and the only operator involved is the standard concatenation on words. As mentioned above, HMSCs can also be seen as particular cases of partial order automata: one simply needs to relabel transitions with the partial order associated with the corresponding MSCs, use as selection function a function that memorizes the last event on each process, and as unique operator the operator that connects for each process this last event to the next occurrence of the minimal event on the same process. In the other way around, observation functions can be applied to POA in a similar way as done for HMSCs: an observation function $\mathcal{O}$ can be applied on a partial order and for a POA $\mathcal{A}, \mathcal{O}(\mathcal{A})=\left\{\mathcal{O}(L) \mid L \in \mathcal{F}_{\mathcal{A}}\right\}$. According to these remarks, the undecidability results for HMSCs immediately extend to POA:

Proposition 4.8. Let $\mathcal{A}, \mathcal{A}_{1}, \mathcal{A}_{2}$ be POA, and let $\mathcal{O}_{1}, \mathcal{O}_{2}$ be two observation functions. Then the inclusion problems $\mathcal{F}_{\mathcal{A}_{1}} \subseteq \mathcal{F}_{\mathcal{A}_{2}}$ and $\mathcal{O}_{1}(\mathcal{A}) \subseteq \mathcal{O}_{2}(\mathcal{A})$ are undecidable.

\subsection{Threaded and locally synchronized partial order automata}

Most of formal properties of HMSCs are undecidable, and NI is no exception. However, decidable subclasses of HMSCs have been identified. Locally synchronized HMSCs have regular linearization languages [Alur and Yannakakis 1999]. Hence inclusion of a regular language, or comparison of HMSC linearizations are decidable problems for locally synchronized HMSCs. It is then reasonable to consider a similar approach for partial order automata and identify subclasses on which comparison of covering relations is decidable. One of the factors that yields decidability in HMSCs is very often the fact that orderings are organized as processes. We can not have similar notions of processes in partial order automata, that only assemble occurrences of labeled events. However, we can use the fact that orders in $\mathcal{F}_{\mathcal{A}}$ are generated as compositions from a finite set of patterns to characterize subclasses of partial order automata where events can be grouped and ordered according to common characteristics, and generate orders with cliques of bounded width.

Definition 4.9 (Threaded POA). A partial order automaton is threaded if for every path $\rho$ of $\mathcal{A}$ containing at least two occurrences of some order $O=(E, \leq, \alpha)$ and every event $e \in E$, we have $e^{(i)}<_{\rho} e^{(i+1)}$ for any two consecutive occurrences of $O$.

The intuition behind threaded POA is that in orders generated by a threaded POA $\mathcal{A}$, the size of cliques (sets of concurrents events) is bounded by a factor of the size of the alphabet labeling events. This allows to organize orders of $\mathcal{F}_{\mathcal{A}}$ in threads, in a similar way as events are localized on processes in HMSCs. This property is essential to be able to decide, for instance, isomorphism of partial order families, as it bounds the degree of the covering relation (an event can only have a bounded number of successors). Consider the example of Fig. 9. Clearly, due to the use of operator $\otimes_{2}$ that creates a 'synchronization barrier', every occurrence $e_{i}$ of some event $e$ in $O_{1}$ or $O_{2}$ precedes the next occurrence $e_{i+1}$ of the same event in any order $O_{\rho}$ associated with a path $\rho$ of $\mathcal{A}$. One can notice that the composition operator $\circ$ of HMSCs immediately grants threaded partial order automata, as MSCs are composed processwise, and hence two successive occurrences of the same event in two occurrences of an MSC are necessarily ordered.

THEOREM 4.10. Given a partial order automaton $\mathcal{A}$ with selection function MaxEvt, one can decide if $\mathcal{A}$ is threaded. Furthermore this problem is in co-NP.

Proof. A path satisfying the property of Def. 4.9 is said to be threaded. We first show that is it sufficient to consider elementary cycles of $\mathcal{A}$ extended by one transition to decide whether a POA is threaded. Consider an accessible cycle $\rho=q \stackrel{O_{1}}{\longrightarrow} q_{1} \ldots \stackrel{O_{k}}{\longrightarrow} q$ of $\mathcal{A}$ and the path $\rho^{\prime}=\rho .\left(q, O_{1}, q_{1}\right)$ that extends $\rho$ with one single transition. We call such a path an elementary sequence. Obviously, if 
there is an event $e$ in $O_{1}$ such that $e^{(1)} \not e^{(2)}$, then any path of $\mathcal{A}$ that ends with $\rho^{\prime}$ is not threaded, and hence $\mathcal{A}$ is not threaded.

Conversely, suppose that all elementary sequences of $\mathcal{A}$ are threaded, but that one can find a path $\rho$ of $\mathcal{A}$ that is not threaded. That is, $\rho$ is of the form $\rho_{1} \cdot\left(q, O_{1}, q^{\prime}\right) \cdot \rho_{2} \cdot\left(q, O_{1}, q^{\prime}\right) \rho_{3}$, and is such that some occurrence $e^{(i)}$ in the $i^{\text {th }}$ occurrence of $O_{1}$ does not precede the $(i+$ $1)^{t h}$ occurrence of $e$ in the $(i+1)^{t h}$ occurrence of $O_{1}$. Clearly, the sequence of transitions $\left(q, O_{1}, q^{\prime}\right) \cdot \rho_{2} \cdot\left(q, O_{1}, q^{\prime}\right)$ is not an elementary sequence, otherwise one would have $e^{(i)} \leq e^{(i+1)}$. However, $\left(q, O_{1}, q^{\prime}\right) \cdot \rho_{2} \cdot\left(q, O_{1}, q^{\prime}\right)$ is obtained by insertion of elementary cycles in an elementary sequence $\rho_{e l}=\left(q, O_{1}, q^{\prime}\right) \cdot \rho_{e l}^{\prime} .\left(q, O_{1}, q^{\prime}\right)$ starting and ending with transition $\left(q, O_{1}, q^{\prime}\right)$. In $\rho$, we have $e^{(1)} \leq e^{(2)}$, that is, there exists a causal chain $e^{(1)} \prec f_{1} \prec \ldots f_{k} \prec g_{1} \prec \ldots \prec g_{k^{\prime}} \prec$ $h_{1} \prec \ldots \prec h_{k^{\prime \prime}} \prec e^{(2)}$, where $f_{1}, \ldots, f_{k}$ are events of $O_{1}^{(1)}, g_{1}, \ldots, g_{k^{\prime}}$ are events of $O_{\rho_{e l}^{\prime}}$, and $h_{1}, \ldots, h_{k^{\prime \prime}}$ are events of $O_{1}^{(2)}$. Consider insertion of an elementary sequence by replacing transition $\left(q_{s}, O_{2}, q_{s+1}\right)$ by an elementary sequence $\rho_{s}=\left(q_{s}, O_{2}, q_{s+1}\right) \ldots\left(q_{s}, O_{2}, q_{s+1}\right)$ in $\rho_{e l}^{\prime}$. Then if there exists no event of $\mathrm{O}_{2}$ in the causal chain from $e^{(1)}$ to $e^{(2)}$, then the causal chain is preserved by replacement of one transition by this elementary sequence. Now, supposing that $\mathrm{O}_{2}$ contains a set of events $g_{t} \prec \ldots g_{t^{\prime}}$ of the causal chain, we still have $e^{(1)} \leq g_{t}^{(1)}$ in $O_{\rho_{s}}$. Similarly, we have $g_{t}^{(1)} \leq g_{t^{\prime}}^{(1)}$, and due to properties of elementary sequences, we have $g_{t^{\prime}}^{(1)} \leq g_{t^{\prime}}^{(2)}$. Now, as the selection function recalls last occurrences of events, and uses the same operators that depend only on chosen transitions, we will have $g_{t^{\prime}}^{(2)} \leq h_{1} \leq \ldots e^{(2)}$. Similar reasoning holds when inserting several occurrences of elementary sequences between two occurrences of $O_{1}$. As all paths containing two consecutive occurrences of some order can only be obtained by such insertions, this allows to conclude that $\rho$ is threaded, which contradicts our starting hypothesis. Hence $\mathcal{A}$ is threaded iff all its elementary sequences are threaded.

Now, let us consider the complexity part. Finding an acyclic path $\rho$ containing twice transitions $\left(q_{1}, O, q_{2}\right)$ can be done non-deterministically in polynomial time, by choosing non-deterministically a path starting from $q_{2}$, and stopping as soon as some transition was already encountered, or when reaching the second occurrence of transition $\left(q_{1}, O, q_{2}\right)$. Followed path are of length at most $|\longrightarrow|$. Appending an order to an existing one and maintaining a set of selected events can be done in polynomial time, as it suffices to add a bounded number of elements (events and covering relation). Denoting by $m$ the maximal size of an order in $\mathbb{L}$, each step hence adds at most $m$ events and $|\mathbb{L}| \times m^{2}$ elements to the covering relation built so far. For a chosen event $e$, one can maintain during construction of the order a set $S$ of at most $|\mathbb{L}| . m$ events that are both in the set of events kept by the selection function, and successors of $e$. Then, if one ends with a second occurrence of $\left(q_{1}, O, q_{2}\right)$, it is easy to check that $e^{(2)}$ is a successor of some event of $S$.

Our overall objective when defining POA is to provide tools to compare partial order families, and in particular HMSC projections. It is well known that locally synchronized HMSCs have regular linearization languages, and as a consequence, many properties are decidable for this subclass of HMSCs. Note however that most of results provided for HMSC rely on properties of their linearizations. When a HMSC has a regular linearization language, the language of its projection onto a subset of events is also regular. However, as shown in the example of Fig. 4, considering linearizations of a system in not always sufficient to characterize information leaks. We will hence rely on a subset of POA, that have a regular linearization language, but more interestingly, for which isomorphism of generated partial order families is decidable. Being threaded is not a sufficient condition for a POA to define a regular language. Inspired by the class of locally synchronized HMSCs, we define an appropriate syntactic class of POA that have regular languages. Locally synchronized HMSCs rely on properties of communication among processes in cycles. POA do not possess this notion of process, but in threaded POA, ordering among events of the same kind replace this total ordering among events located on the same process. We hence rely on properties of a commutation graph (instead of communication graphs in HMSCs) to define locally synchronized POA. 
Definition 4.11. Let $O=(E, \leq, \alpha)$ be a partial order, and $\rho$ be a path such that $O=O_{\rho}$. The commutation graph of $\rho$ is a graph $C G(\rho)=(E, \rightsquigarrow)$ where $(e, f) \in \rightsquigarrow$ iff

- $e \leq_{\rho} f$ ( $e$ precedes $f$ in $O_{\rho}$ ),

- $e^{(1)} \leq_{\rho . \rho} f^{(2)}$ (the first occurrence of $e$ precedes the next occurrence of $f$ ).

A POA $\mathcal{A}$ is locally synchronized if it is threaded, and for each cycle $\rho$ of $\mathcal{A}, C G(\rho)$ is strongly connected.

Consider again the example of Fig. 9. We already know that this POA is threaded. Furthermore, the synchronization barrier imposed by operator $\otimes_{2}$ guarantees that every occurrence of an event $e$ in $O_{1} \otimes O_{2}$ either precedes either the occurence of en event $f$ in $O_{1} \otimes O_{2}$ (this is for instance the case for events $e_{1}, e_{2}$ in the example at the right of the figure), or precedes the next occurrence of this event (this is for instance the case for events $e_{1}$ and $e_{9}$ in the example). This property applies for any pair of events, and also for order $O_{2} \otimes O_{1}$, so the POA of Fig. 9 is locally synchronized.

THEOREM 4.12. Let $\mathcal{A}$ be a threaded POA with a selection function $\Pi$ that memorizes a bounded number $K$ of events. Then one can effectively decide if $\mathcal{A}$ is locally synchronized.

PROOF. (Sketch) Existence of disconnected communication graphs can be proved on cycles that contain at most one occurrence of each transition. Indeed, for a selected pair of events $e, f$ in such cycles, as considered automata are threaded, insertion of another elementary cycle simply extends the length of causal chains and does not change connectedness of $e^{(1)}, f^{(1)}, e^{(2)}, f^{(2)}$. It then suffices to detect these cycles, build their commutation graph and check that these graphs are connected.

\subsection{Finitely decomposable observation functions}

So far, we have identified a class of threaded and locally synchronized POA, that appear as good candidates to represent some observations of locally synchronized HMSCs. It then remains to show that this is the case. For this, we proceed in two steps: we first define properties of observations functions that guarantee that observation of families of MSCS obtained by sequential composition from a predetermined set of MSCs $\mathcal{M}$ can be effectively represented by POA. Namely, we require the observation of orders in $\mathcal{M}^{\circ *}$ to be expressible as compositions of observations of MSCs from $\mathcal{M}$ with a finite set of operators, and we require existence of a function that can choose the right operator to assemble orders at each moment of the sequence.

Definition 4.13. An observation function $\mathcal{O}$ is decomposable w.r.t. a set of MSCs $\mathcal{M}$ iff there exists a finite set of operators $O P S=\left\{\otimes_{1}, \ldots, \otimes_{k}\right\}$ and a function $\Psi: \mathcal{M}^{\circ *} \rightarrow$ OPS such that for every pair of MSCs $M_{1}, M_{2}$ in $\mathcal{M}^{\circ *}, \mathcal{O}\left(M_{1} \circ M_{2}\right)=\mathcal{O}\left(M_{1}\right) \otimes_{\Psi\left(M_{1}\right)} \mathcal{O}\left(M_{2}\right)$, where $\otimes_{\Psi\left(M_{1}\right)}=$ $\Psi\left(M_{1}\right)$.

Decomposability of an observation function w.r.t. to a set of MSCs guarantees that the set of operations needed to assemble the observations of two MSCs and obtain the observation of the concatenation of these MSC is finite, and that the operation to apply only depends on the order observed so far. This is a first step towards some form of compositionality for observations. This is however not sufficient to build incrementally an observation of a HMSC, as one may still need unbounded memory to assemble two observations.

Definition 4.14. An observation function $\mathcal{O}$ is finitely decomposable iff it is decomposable, and

(1) there exists a bound $c \in \mathbb{N}$ such that for every sequence of MSCs $M_{1}, \ldots, M_{k}$, and $\forall j \in 1 . . k$, denoting by $\left|<_{i . . j}\right|$ the size of the covering of $\mathcal{O}\left(M_{i} \circ \ldots \circ M_{j}\right)$, we have $\mid<_{1 . . k} \backslash\left(<_{1 . . j}\right.$ $\left.\cup<_{j . . k}\right) \mid<c$. Intuitively, the events in every MSC $M_{j}$ are connected to a bounded number of predecessors and successors, regardless of the index $j$.

(2) there exists a bound $m$ and a selection function $\Pi$ such that for every sequence of MSCs, $M_{1}, \ldots, M_{k}$, for every $(i, j)$ with $1<i<j<k$, 
(a) $\Psi$ is regular, i.e., there exists a deterministic finite state machine $\mathcal{B}_{\Psi}$ that reads sequences of MSCs and associates an operator with every state.

(b) $\Pi\left(\mathcal{O}\left(M_{1}\right) \otimes \cdots \otimes \mathcal{O}\left(M_{i}\right)\right)$ is a set $\mathrm{Mem}_{i}$ of size at most $m$ containing events in $\mathcal{O}\left(M_{1}\right) \otimes$ $\cdots \otimes \mathcal{O}\left(M_{i}\right)$

(c) $<_{i, i+1} \subseteq \operatorname{Mem}_{i} \times E_{i+1}$ (the memorized events are sufficient to build the ordering from events in $O_{1} \ldots O_{i}$ to events in $\left.O_{i+1}\right)$,

(d) $\Pi\left(\mathcal{O}\left(M_{1}\right) \otimes \cdots \otimes \mathcal{O}\left(M_{j}\right)\right)$ is a set $\mathrm{Mem}_{j}$ of size at most $m$ containing events in $\mathcal{O}\left(M_{1}\right) \otimes$ $\cdots \otimes \mathcal{O}\left(M_{j}\right)$ such that $M_{e m} \cap O_{1} \ldots O_{i} \subseteq$ Mem $_{i}$

Intuitively, for finitely decomposable observation functions with memorization function $\Pi$, this function keeps in memory only a bounded number of events that need to be used later along observation of a sequence, and the computation of the memory is compositional, in the sense that it removes useless events from memory at previous steps, and adds new events that will be used later.

THEOREM 4.15. For every HMSC $H=\left(N, \longrightarrow, \mathcal{M}, n_{0}, F\right)$, and every finitely decomposable observation function $\mathcal{O}($ w.r.t. $\mathcal{M})$, one can build a $P O A \mathcal{A}_{H, \mathcal{O}}$ that recognizes $\mathcal{O}(H)$.

Proof. For a given HMSC $H=\left(N, \longrightarrow, \mathcal{M}, n_{0}, F\right)$ we build the finite partial order automaton $\mathcal{A}_{H, \mathcal{O}}=\left(Q, \longrightarrow{ }^{\prime}, \mathcal{O}(\mathcal{M}), q_{0}, F^{\prime}, \Lambda, \Pi\right)$. We define $Q=\left\{n_{0}\right\} \cup N \times O P S$, where $O P S$ is the set of operators used by the finitely decomposable observation function $\mathcal{O}$. We set $\longrightarrow$ ' as the set of triples of the form $\left((n, o p), \mathcal{O}(M),\left(n^{\prime}, o p^{\prime}\right)\right)$ such that $\left(n, M, n^{\prime}\right) \in \longrightarrow$ and there exists a path $\rho=n_{0} \stackrel{M_{1}}{\longrightarrow} n_{1} \ldots \stackrel{M_{k}}{\longrightarrow} n$ of $H$ such that $\Psi\left(M_{1} \circ \ldots M_{k}\right)=o p$ and $\Psi\left(M_{1} \circ \ldots M_{k} \circ M\right)=o p^{\prime}$. As $\Psi$ is regular, $\longrightarrow$ is finite and can be built inductively. Last, $\Lambda: Q \times \mathcal{O}(\mathcal{M}) \times Q \rightarrow O P S$ associates operator $o p$ with every transition $t=\left((n, o p), \mathcal{O}(M),\left(n^{\prime}, o p^{\prime}\right)\right) \in \longrightarrow^{\prime}$, and $\Lambda\left(\left(n_{0}, O_{i}, n^{\prime}\right)\right)=i d$, that is an observation starting from the initial node of the HMSC simply copies the observation of the first MSC recognized from the initial node of $H$.

Proposition 4.16. For every HMSC $H$, observation functions $\mathcal{O}^{V}, \mathcal{O}_{\backslash C}^{V}, \mathcal{O}^{p}$ are finitely decomposable, with bounds $m \leq|\mathbb{P}|^{2}$ and $c \leq|\mathbb{P}|^{3}$.

Proof. (Sketch) We build this proof on the result of [Genest et al. 2003], that shows that projections of HMSCs can be recognized by finite partial order automata. These automata memorize events that can still have a successor in the projected covering relation, and use a single composition operator that connects the projection of a newly observed MSC to memorized events (whence finite decomposability of the function that associates an operator to sequences of MSCs). As the set of events to memorize is always finite, as shown in [Genest et al. 2003], one can design a POA with finite memory selection function that recognizes $\mathcal{O}^{V}(H)$. The proof for $\mathcal{O}_{\backslash C}^{V}(H)$ and $\mathcal{O}^{p}(H)$ is similar.

A consequence of this proposition is that one can build partial order automata that generate $\mathcal{O}^{V}(H), \mathcal{O}_{\backslash C}^{V}(H), \mathcal{O}^{p}(H)$. One can also notice that automata that recognize projections of HMSCs are threaded, since with the composition operators used, the $n^{\text {th }}$ event on a process necessarily precedes the $n+1^{t h}$ event on the same process. Now, this does not mean that inclusion properties are decidable. We have to consider subclasses of partial order automata, and then check that observations fall into these subclasses.

\section{INTERFERENCE DETECTION ALGORITHMS}

In this section, we prove that non-interference is decidable for locally synchronized HMSCs. To check an inclusion problem for an HMSC $H$, and subsequently check non-interference, one needs to compare runs in $\mathcal{A}_{\mathcal{O}_{1}, H}$ and $\mathcal{A}_{\mathcal{O}_{2}, H}$ for two suitable observation functions $\mathcal{O}_{1}, \mathcal{O}_{2}$. A run $\rho_{2}$ of $\mathcal{A}_{\mathcal{O}_{2}, H}$ is compatible with a run $\rho_{1}$ of $\mathcal{A}_{\mathcal{O}_{1}, H}$ if $O_{\rho_{1}}$ is a prefix of $O_{\rho_{2}}$ (i.e., one can find a matching function $h$ sending events of $O_{\rho_{1}}$ onto $O_{\rho_{2}}$, as in the definition of prefix in section 2). Notice that there can be several runs of $\mathcal{A}_{\mathcal{O}_{2}, H}$ (possibly an infinite number of them) that are compatible with 
a chosen run $\rho_{1}$. However, as soon as partial order automata are threaded, we can give a finite representation for sets of runs that comply with a finite order.

\subsection{Minimal explanations and unfoldings of POA}

One important fact with HMSCs is that if a run is compatible with a given observation, then extending this run with an additional transition still produces a compatible run. Similarly, one can consider cycles that have no observable effect as implicit. These properties still hold for threaded POA, and can be used to find minimal and finite sets of explanations.

Definition 5.1 (Minimal explanations). Let $O, O^{\prime}$ be LPOs, let Mem be a subset of events of $O^{\prime}$, and let $\mathcal{A}$ be a threaded POA with finite memory function $\Pi$ and let $q$ be a state of $\mathcal{A}$. The set of minimal explanations of $\mathcal{A}$ compatible with $O$ starting from $O^{\prime}$, Mem, $q$ is the set of all shortest paths of the form $\rho=\left(q, O_{1}, q_{1}\right) \ldots\left(q_{k-1}, O_{k}, q_{k}\right)$ of $\mathcal{A}$, starting from $q$ such that $O \sqsubseteq O^{\prime} \otimes O_{1} \otimes \cdots \otimes O_{k}$.

Considering shortest paths is one essential requirement to have a finite representation for the set of paths of $\mathcal{A}$ that have a particular order $O$ as prefix. Hence, if $O$ is already a prefix of $O_{\rho}$, we do not consider paths of the form $\rho . \rho^{\prime}$. This is however not sufficient to obtain a finite representation of paths embedding $O$ : a set of minimal explanations can still be infinite. Indeed, consider an order $O$ with only two events $a \leq a^{\prime}$. Then $O$ could be a prefix of any order of the form $O_{1}\left(\otimes O_{2}\right)^{k} \otimes O_{3}$ where $O_{1}$ contains $a, O_{3}$ contains $a^{\prime}$, and $O_{2}$ only events that are not causally related to occurrences of $a$ or $a^{\prime}$. However, such iterations can be handled. We reuse ideas from [Hélouët et al. 2014] where a finite unfolding of an HMSC is built to perform diagnosis from a partial order observation, and an abstraction technique introduced in [Alur and Yannakakis 1999] to represent finitely sequences of MSCs that are partially executed. Let us first build a finite representation for this set of paths.

Starting from POA $\mathcal{A}=\left(Q, \longrightarrow, \mathbb{L}, q_{0}, F, \Lambda, \Pi\right)$ and LPO $O$, we build inductively a POA $\mathcal{B}$, where states and transitions are obtained by unfolding $\mathcal{A}$, and remembering after each transition the part of $O$ that is a prefix of a path ending in this state, and the memorized events. States are hence of the form $\left(q, \operatorname{Mem}_{q}, E_{q}\right.$, ), where $q$ is a state of $\mathcal{A}, \operatorname{Mem}_{q}$ is a description of memorized events (a subset containing events from Mem - the initial memory contents- and newly generated events), $E_{q}$ is a subset of events of $E_{O}$. There is a transition from $\left(q, \operatorname{Mem}_{q}, E_{q},\right)$ to $\left(q^{\prime}, \operatorname{Mem}_{q}^{\prime}, E_{q}^{\prime}\right)$ labeled by $O_{i}$ iff there exists a transition $\left(q, O_{i}, q^{\prime}\right)$ in $\mathcal{A}$, and:

- $E_{q} \neq E_{O}(O$ was not already regognized $)$

- $\mathrm{Mem}_{q}^{\prime}=\Pi\left(\mathrm{Mem}_{q} \otimes O_{i}\right)$, where $\otimes=\Lambda\left(q, O_{i}, q^{\prime}\right)$,

- $E_{q}^{\prime}$ is the maximal subset of events of $E_{O}$ that contains $E_{q}$ and such that $O_{\mid\left(E_{q}^{\prime} \backslash E_{q}\right) \cup M e m_{q}} \sqsubseteq$ $\operatorname{Mem}_{q} \otimes O_{i}$.

Intuitively, appending $O_{i}$ to already built paths allows us to embed a larger part of $O$ in the recognized order. We define $\Lambda^{\prime}\left((q, \mathrm{Mem}, E), O_{i},\left(q^{\prime}, \mathrm{Mem}^{\prime}, E^{\prime}\right)\right)=\Lambda\left(q, O_{i}, q^{\prime}\right)$ and $\Pi^{\prime}=\Pi$. During this construction, we may create loops that do not change the recognized part of $O$, nor the memory contents. States of the form $\left(q, \operatorname{Mem}_{q}, E_{O}\right)$ have no successor $(O$ is a prefix of orders generated along all paths ending in this state) and are called final states. The construction can be performed inductively and stops when no new state is discovered. If the memory selection function of $\mathcal{A}$ memorizes only a finite number of events, and if $\mathcal{A}$ is threaded (which guarantees that the set of paths of $\mathcal{A}$ to explore to find the next occurrence of some action is bounded), then this construction terminates, and for every $O^{\prime} \in \mathcal{F}_{\mathcal{B}}, O \sqsubseteq O^{\prime}$.

We can then extract from $\mathcal{B}$ a finite set of sequential representations for the minimal explanations of $O$ as follows: it is the set of acyclic paths from $q_{0}$ to a final state, decorated with connected components for which transitions do not change the memory contents nor the part of $O$ discovered so far. We call these transitions silent transitions: They are labeled by orders with events that might appear in larger orders containing $O$, but are not mandatory to find a path $\rho$ such that $O \sqsubseteq O_{\rho}$. Similarly, one can find minimal explanations from any state $q$ starting with an already recognized order $O^{\prime}$ and memory contents $\mathrm{Mem}$. 
As $\mathcal{A}$ is threaded, for every transition $t=\left((q, M e m, E), O_{i},\left(q^{\prime}, \mathrm{Mem}^{\prime}, E^{\prime}\right)\right)$, one can find which events of $O_{i}$ are used to witness embedding of $O_{\mid\left(E_{q}^{\prime} \backslash E_{q}\right) \cup M e m_{q}}$ into $M e m \otimes O_{i}$ (i.e., are used to build a matching from $O$ to $O^{\prime} \otimes O_{1} \ldots O_{i}$ ). Once $\mathcal{B}$ is computed, we can compute a partial map $h_{\mathcal{B}}()$ that associates with an event along each transition $\left(q, O_{i}, q^{\prime}\right)$ the event in $E_{O}$ to which it corresponds. We say that an event $e$ is marked if $h_{\mathcal{B}}(e)$ is defined, and denote by Marked $(t) \subseteq E_{O_{i}}$ the set of events marked in a transition $t=\left((q, M e m, E), O_{i},\left(q^{\prime}, M_{e m}^{\prime}, E^{\prime}\right)\right)$. Note that it is not always the case that $\operatorname{Marked}(t)=E_{O_{i}}$ : when the transition reaches a final state of $\mathcal{B}$, a suffix of $O_{i}$ may not be used to witness an embedding of $O$. Similarly, $O_{i}$ can contain unmatched events located on parallel threads that will never be used to witness embedding of $O$ along current path. We say that transition $t$ is incompletely marked if $\operatorname{Marked}(t) \neq E_{O_{i}}$.

\subsection{Checking inclusion for POA}

Now, let $\mathcal{A}_{1}$ and $\mathcal{A}_{2}$ be two partial order automata. Let $O=O_{1} \otimes O_{2} \otimes \ldots O_{n}$ be an order generated along a path $\rho_{1}$ of $\mathcal{A}_{1}$, and let $\mathcal{B}$ be the minimal unfolding of $\mathcal{A}_{2}$ starting from $q_{2,0}$ (the initial state of $\mathcal{A}_{2}$ ) with empty order and memory. Let $\rho_{2,1}, \ldots \rho_{2, k}$ be explanations provided by $\mathcal{B}$ ending on final states $q_{1}, \ldots q_{k}$. These explanations $\rho_{2,1}, \ldots \rho_{2, k}$ are paths decorated with silent connected components, hence they are partial order automata. Let $h_{1}, \ldots, h_{k}$ be the mappings associated with transitions in $\rho_{2,1}, \ldots \rho_{2, k}$, that link every event along path $\rho_{2, i}$ to the corresponding event of $O$, and let $M r k_{1}, \ldots, M r k_{k}$ denote the set of marked events along each path and let $\mathcal{B}_{i}$ denote the partial order automaton obtained by adding to $\rho_{2, i}$ all transitions of $\mathcal{A}$ that are accessible from $q_{i}$. Then, $O \otimes O_{n+1}$ is a prefix of some order generated by $\mathcal{A}_{2}$ iff it is a prefix of an order generated by one of the $\mathcal{B}_{i}$ 's. Hence, $O \otimes O_{n+1}$ is a prefix of some order generated along a path $\rho_{2}$ of $\mathcal{A}_{2}$ if $\rho_{2}$ can be decomposed as $\rho_{2}=\alpha_{1} \beta_{1} \alpha_{2} \ldots \alpha_{k} \cdot \gamma$, where $O \sqsubseteq O_{\alpha_{1} \ldots \alpha_{k}}, \alpha_{1} \ldots \alpha_{k}$ is a path of $\mathcal{A}$ ending in some state $q_{\alpha}$ with memory $\mathrm{Mem}_{\alpha}, \beta_{i}$ are finite sequences of silent transitions allowed between $\alpha_{i}$ and $\alpha_{i+1}$, and $\gamma$ is a finite sequence of transitions obtained from an unfolding of $\mathcal{A}_{2}$ to check inclusion of $O_{n+1}$ starting from state $q$ with memory $\mathrm{Mem}_{\alpha}$, and from the restriction of order $O_{\alpha_{1} \beta_{1} \alpha_{2} \ldots \alpha_{k}}$ to unmarked events. However, the converse operation is more interesting. Starting from an order $O$, and an explanation $\rho$ (a sequence of transition with silent connected components attached to some states) as we add only a finite number of events and as $\mathcal{A}_{2}$ is threaded, one can hence compute all possible explanations for $O \otimes O_{n+1}$ by choosing adequate $\beta_{i} s$ in connected components of $\rho$ and then computing $\gamma$ as an unfolding of $\mathcal{A}$ starting from the final state of $\rho$.

As proposed in [Alur and Yannakakis 1999] we can go further, and memorize only subsequences of each path that contain incomplete transitions, and the connection between these subsequences. Let $\mathcal{B}_{i}$ be the automaton associated with an explanation as above, and suppose that it starts with a single transition $t=\left(q, O_{1}, q^{\prime}\right)$ (i.e., its initial state is not attached to a silent strongly connected component) such that events of $t$ are all marked. Then $O \otimes O_{n+1}$ is a prefix of some order generated by $\mathcal{A}_{2}$ iff $O \otimes O_{n+1} \backslash h_{i}\left(E_{O_{1}}\right)$ is a prefix of $\mathcal{B}_{i} \backslash\{t\}$ with initial state $q^{\prime}$. Hence, one can safely forget initial transitions which are all marked. Last, $O \otimes O_{n+1}$ is a prefix of some order generated by $\mathcal{A}_{2}$ iff $O_{n+1}$ is a prefix of the projection of some $O_{\rho}$ where $\rho$ is a path of some $\mathcal{B}_{i}$ on its unmarked events. This means that one can simply memorize incompletely marked transitions, silent connected components, final states of all $\mathcal{B}_{i} s$ and still check that appending a particular order $O_{n+1}$ preserves the inclusion proved so far. Starting from an explanation $\rho_{2, i}$ we denote by $\operatorname{Prune}\left(\rho_{2, i}\right)$ the sequence of incompletely marked transitions and connected components obtained from $\rho_{2, i}$. As extension of an explanation only uses connected components or appends orders at the end of the explanation, one can compute a new explanation from a pruned explanation. For an explanation $\rho$ proving that an order $O$ is a prefix of some order of $\mathcal{A}_{2}$, we denote by $\operatorname{Succ}\left(\rho, O_{n}\right)$ the set of explanations obtained this way for $O \otimes O_{n}$.

This immediately gives the idea of algorithm 1 below to compare two partial order automata $\mathcal{A}_{1}$ and $\mathcal{A}_{2}$. The algorithm follows paths of $\mathcal{A}_{1}$, by remembering a set of selected events in memory and the last state visited in $\mathcal{A}_{1}$, and on the other side, it maintains a set of pruned explanations of $\mathcal{A}_{2}$ that are compatible with the followed paths. At each step of the construction, when choosing a new 
transition $t=\left(q, O_{n}, q^{\prime}\right)$ from $\mathcal{A}_{1}$, i.e., extending some path $\rho_{1}$, we ensure that $O_{\rho_{1} . t}$ is a prefix of an order for at least one explanation. Note however that pruning does not guarantee finiteness of the memorized information in general. The algorithm returns false if it can find a path $\rho_{1}$ that has no explanation in $\mathcal{A}_{2}$, and true if all possible configurations have been explored.

The set of configurations to explore can grow arbitrarily, and nothing guarantees that the algorithm terminates in general. However, locally synchronized POA produce only regular sets of linearizations, and describe behaviors in which no process can repeat a behavior, i.e., iterate a behavior described in a cycle of the POA, as long as the preceding occurrence of this cycle is not terminated by other contributing processes.

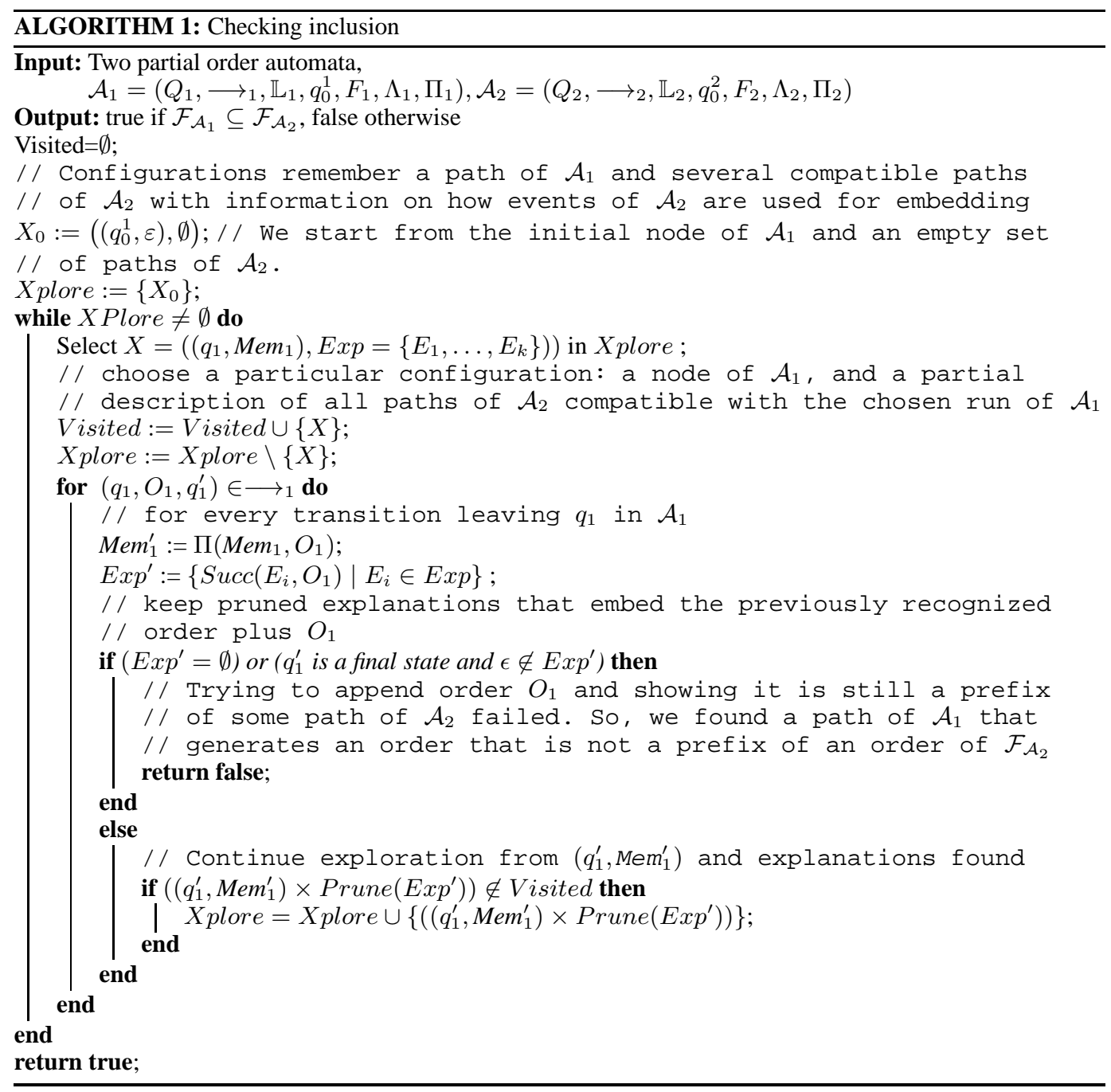

THEOREM 5.2. If $\mathcal{A}_{1}$ and $\mathcal{A}_{2}$ are locally synchronized POA, then the order inclusion algorithm terminates. 
Proof. Suppose that at some stage, an explanation $\rho_{2}$ obtained when recognizing an order $O_{\rho, 1}$ contains more than $|\mathcal{A}|$ states from which some cycle $\beta$ coming from a silent connected component can be appended. In other words, $\rho_{2}$ is an explanation for paths of the form $\rho=\rho_{2,1} \cdot \rho_{2,2} \ldots \rho_{2, k}$, where occurrence of a cycle can be inserted between each pair $\rho_{2, i}, \rho_{2, i+1}$. As it is of size greater that $\mathcal{A}$, then $\rho$ necessarily contains a cycle. As inserting $\beta$ is optional to explain $O_{\rho, 1}$ we know that inserting the contents of this cycle does not change the set of observed events nor causalities, i.e. $O_{\beta}$ is an LPO that is completely concurrent with $O_{\rho}$. However, if $\beta$ commutes with elements of a path of size greater than $|\mathcal{A}|$, then $\mathcal{A}$ is not locally synchronized. So, all possible insertion of cycles in an acyclic explanation can occur between transitions of a path located in a suffix of this path of size at most $|\mathcal{A}|$. Hence, there are less than $|\mathcal{A}|$ silent cycles in any explanation. Now in a given path $\rho$ of $\mathcal{A}_{2}$, if the $i^{\text {th }}$ occurrence of an event in a particular order $O_{j}$ is marked, then the preceding occurrences are also marked. As we do not add any transition to pruned path sequences that end with unmarked order (otherwise these sequences would not be minimal) in a path of size greater than $|\mathcal{A}|^{2}$.max where max is the size of the largest order in $\mathcal{A}_{2}$, there is necessarily a sequence of transitions carrying only marked events. Hence, there is only a finite number of configurations for subsequences describing the yet unexplained part of a path followed in $\mathcal{A}_{1}$ and the matching paths of $\mathcal{A}_{2}$. So, the algorithm terminates.

PROPOSITION 5.3. If $H$ is a locally synchronized HMSC, then $\mathcal{A}_{H, \mathcal{O}^{V}}$ and $\mathcal{A}_{H, \mathcal{O}^{V}}$ are locally synchronized partial order automata.

PROOF. If $H$ is locally synchronized, then for any cycle $\rho$, and pair of events $e, f$ in $M_{\rho}$, we have $e^{(1)} \leq f^{(2)}$ and $f^{(1)} \leq e^{(2)}$ in $M_{\rho} \circ M_{\rho}$. As $\mathcal{O}^{V}$ is simply a projection, for any pair of events with labels in $V, e^{(1)}, f^{(1)}, e^{(2)}, f^{(2)}$ are ordered similarly. Hence $\mathcal{A}_{H, \mathcal{O}^{V}}$ is locally synchronized. Not every cycle of $H$ becomes a cycle of $\mathcal{A}_{H, \mathcal{O}_{C}^{V}}$, as $\mathcal{O}_{\mid C}^{V}$ may force to remove more events on transitions than a simple projection. However, cycles of $\mathcal{A}_{H, \mathcal{O}_{Y C}^{V}}$ are also obtained from cycles of $H$ and labeled by projections, hence $\mathcal{A}_{H, \mathcal{O}^{V}}$ is also locally synchronized.

We then have the obvious following corollary:

COROLLARY 5.4. Non-interference is decidable for locally synchronized HMSCs.

\section{LOCAL AND CAUSAL NON INTERFERENCE}

We now turn to other types of decidable classes, related to regularity. Indeed, inclusion problems become decidable as soon as one can recast the order comparison problem in a regular setting. It is however undecidable whether an HMSC or a partial order automaton has a regular behavior, and one has to rely on syntactic subclasses of the models such as locally-synchronized HMSCs/POA as above to obtain decidability. We show in this section that several HMSC observation functions describing the discriminating power of a single process always define sets of orders that can be recognized by finite (word) automata, regardless of the characteristics of the considered HMSC. In this restricted setting, it is then possible to decide whether a process $p \in \mathbb{P}$ can detect occurrences of confidential actions. As HMSCs explicitly specify distribution of actions on processes, exhibiting the behavior of a fixed process within an HMSC specification is an easy task. In this section, we show that this local setting allows for the definition of two decidable notions of non-interference.

\subsection{Local interference}

Considering the attacker of a system as a single process $p \in \mathbb{P}$, with action labels in some alphabet $\Sigma_{p}=\alpha\left(E_{p}\right)$, we should assume that process $p$ does not execute confidential actions, that is $C \cap$ $\Sigma_{p}=\emptyset$. In a similar way, the observation power of a single process should be restricted to its own events, hence we can safely set $V=\Sigma_{p}$. The definition of non-interference (Def. 3.3) proposed in section 3 can accommodate this particular partition of the alphabet. From now on, we consider this restricted form of non-interference, and call it local non-interference. 
For a single MSC, it is then defined as satisfaction of two inclusion problems, with $\mathcal{O}_{\backslash C}^{V}$ and $\mathcal{O}^{p}$ as observation functions. This property can be verified by checking whether $\uparrow\left(\alpha^{-1}(C)\right) \cap E_{p}=\emptyset$ that is checking if no causal consequence of a confidential action is located on process $p$. Recast in the setting of MSC coloring, this amounts to checking that $p$ is not marked with a black token. As explained in Section 3.3, this can be performed in linear time. We can now look at local interference for HMSCs:

Definition 6.1. Let $H$ be an HMSC over a set of processes $\mathbb{P}$, over alphabet $\Sigma=V \uplus C \uplus N$. Let $p \in \mathbb{P}$ be a process, and $\Sigma_{p}$ be the alphabet of actions located on process $p$, with $V=\Sigma_{p}$. Then $H$ is locally non-interferent w.r.t. process $p$ if $\mathcal{O}_{\backslash C}^{V, \bullet}(H) \equiv \mathcal{O}^{V, \circ}(H)$.

Intuitively, local interference holds when an observer can not distinguish in $\mathcal{F}_{H}$ behaviors that are concatenations of MSCs containing no confidential event, and other behaviors. Consider HMSC $H^{\prime \prime}$ in Fig. 10. $H^{\prime \prime}$ is interferent according to definition 6.1, as observation of ? $m$ or ? $n$ on process $p$ differentiates executions with/without confidential event $c$. However, no event on $p$ is a causal consequence of $c$.

Proposition 6.2. For every HMSC $H$ and every process $p \in \mathbb{P}$, one can build a (partial order) automaton $\mathcal{A}_{H, \mathcal{O}^{p}}$ that recognizes $\mathcal{O}^{p}(H)$. If $V=\Sigma_{p}$, then one can build (partial order) automata $\mathcal{A}_{H, \mathcal{O}}^{V, \bullet}$ and $\mathcal{A}_{H, \mathcal{O}}$,o that recognize respectively $\mathcal{O}_{\backslash C}^{V, \bullet}(H)$ and $\mathcal{O}^{V, \circ}(H)$.

Proof. (Sketch) For any $H$, we can build a finite automaton $\mathcal{A}_{p}(H)$ that recognizes (linearizations of) projections of all MSCs in $\mathcal{F}_{H}$ on $p$. As concatenation of MSCs imposes a total order on events of the same process, these projections are concatenations of finite sequences of events (local projections of MSCs along transitions of $H$ ). Hence $\mathcal{A}_{p}(H)$ has transitions using labels of events located on process $p$, and just needs to remember the transition of $H$ that is recognized (the current MSC under execution), and a bounded integer symbolizing the last event of the current MSC executed by $p$. Similarly, we can design an HMSC $H_{\backslash C}$ where transitions are labeled by MSCs that do not contain confidential events, and hence an automaton $\mathcal{A}_{p}^{\prime}(H)$ that accepts only projections on $p$ of sequences of MSCs with only visible (white) events. Hence $\mathcal{A}_{p}^{\prime}(H)$ recognizes $\mathcal{O}_{\backslash C}^{V, \bullet}(H)$. Last, as $V=\Sigma_{p}$, then $\mathcal{O}^{V, \circ}(H)=\mathcal{O}^{p}(H)$.

Recast in the context of partial order automata, the automata $\mathcal{A}_{H, \mathcal{O}^{p}}$ and $\mathcal{A}_{H, \mathcal{O}_{\backslash C}^{V, \bullet}}$ built in proposition 6.2 are locally synchronized, have finite memory functions (that remember only the last event appended), and a unique composition operator $\circ$ (that assembles sequences of events on process $p$ ). It should be noted that $\mathcal{A}_{H, \mathcal{O}^{p}}$ and $\mathcal{A}_{H, \mathcal{O}_{i}^{V, \bullet}}=\mathcal{A}_{p}^{\prime}(H)$ (and hence also $\mathcal{A}_{H, \mathcal{O}}, \mathrm{O}$ ) are in fact finite word automata, yielding decidability of inclusion and interference.

COROLLARY 6.3. The problem of deciding local interference of an HMSC H with respect to a given process $p \in \mathbb{P}$ is PSPACE-complete.

ProOF. (Sketch) When considering projection on a single process $p$, the automata $\mathcal{A}_{H, \mathcal{O}},{ }_{C}^{V,}$ and $\mathcal{A}_{H, \mathcal{O}}$,o built in proposition 6.2 recognize sequences of events located on process $p$. They are hence standard word automata (where each transition is labeled by a single event), and checking local interference resumes to inclusion of the languages of these automata. (whence the complexity in PSPACE). For the hardness part, we can also show that any regular language inclusion problem can be encoded as a local interference problem.

Local interference is decidable, and describes a situation where a process can discover that the running execution of the system contains or will contain a confidential action. Consider for instance the HMSC Of Fig. 3 with $C=\{g\}$ and its observation on process $r$. Any execution containing an event with label $i$ reveals occurrence of $\mathrm{MSC} M_{4}$, and hence the possibility that $g$ has occurred 
or will occur. However, local interference does not distinguish between a situation where an observation is a causal consequence of some confidential action and a situation where observation and confidential action highlighted by the interference are concurrent. This drawback also occurs in standard language-based interference settings, where causality is represented as interleaving, and one can not decide whether in a word $c . v$ actions $c$ (confidential) and $v$ (visible) are concurrent or not.

\subsection{Causal interference}

We first give a concrete example showing that leaking information in a causal order context may give opportunities for focused security attacks when the confidential event that is detected lays within the causal past of some observation. Nowadays, a lot of attention is devoted to privacy. However, it is well known that users spread a lot of information to visited sites when browsing the web. This information is not always local information (cookies, cache, etc.) that can be erased by users if needed. It can also be information stored elsewhere on the web: $\log s$, forms, etc. . When observation of a causal consequence of a confidential action ( $\mathrm{Mr} X$ has bought a book on commercial site $Y$ ) by an attacker indicates that a confidential operation has occurred, this may also mean that classified information might be available at some vulnerable site (the credit card details of $X$ are stored somewhere on $Y$ 's website). Hence, characterizing interference where confidential actions and observations are causally related, is important.

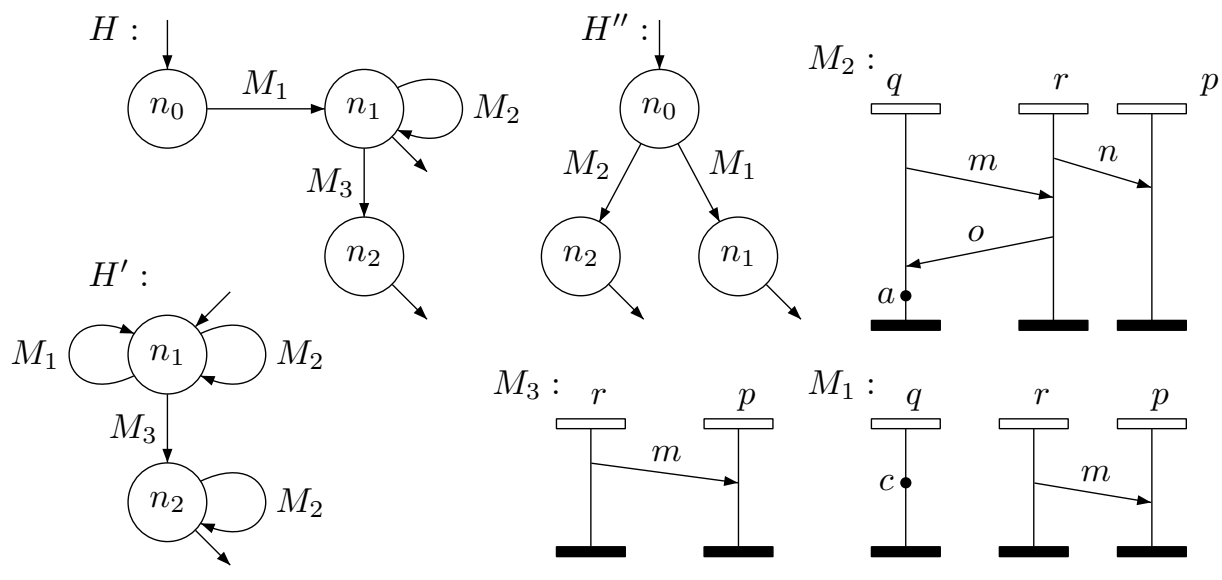

Fig. 10. An interferent HMSC

Example 6.4. Consider the HMSCs $H, H^{\prime}, H^{\prime \prime}$ depicted in Fig. 10, with $C=\{c\}$. The projection of MSCs recognized by $H$ on $p$ is the language ?m. $(? n)^{*} . ? m$. Each word of the form $? m .(? n)^{k} . ? m$ observed by $p$ is the projection of a run with labels $M_{1} \circ M_{2}^{k} \circ M_{3}$. If $k>2$, then the second reception of message $n$ causally depends on confidential event $c$. Hence $H$ is causally interferent. Now, consider HMSC $H^{\prime}$. The projection of executions on process $p$ is the language $(? m+? n)^{*} . ? m . ? n^{*}$. Upon reception of message $m$ followed by two messages $n$ on process $p$ one cannot decide whether and occurrence of action $c$ occurred or not. HMSC $H^{\prime}$ is not causally interferent. Last, HMSC $H^{\prime \prime}$ is not causally interferent (there is no causal dependeny from $c$ to any other event in executions of $H^{\prime \prime}$ ), but it is locally interferent (as defined in def. 6.1).

In the rest of this section, we propose a decidable notion of causal interference (still with respect to a fixed attacker $p \in \mathbb{P}$ ). It emphasizes on causal dependencies between confidential and visible actions of the system. Bearing in mind that a black event located on process $p$ is a consequence of a confidential event, we show that causal dependencies can be discovered by maintaining in states of a HMSC the information on black/white tokens attached to processes. We want to check if a process $p$ can detect whether some confidential action has occurred in the causal past of its observed events. 
In other words, we have to check whether all projections on $p$ of an execution of $H$ that contains a black event, only have equivalent projections that do not contain black events.

Definition 6.5. For an HMSC $H$ and a process $p \in \mathbb{P}, H$ is causally non-interferent with respect to $p$ if for every MSC $M$ in $\mathcal{F}_{H}$ such that $M$ contains a black event on process $p$, there exists another MSC $M^{\prime}$ in $\mathcal{F}_{H}$ such that:

- $M^{\prime}$ contains no black event on process $p$, and

- $\mathcal{O}^{p}(M)=\mathcal{O}^{p}\left(M^{\prime}\right)$

According to definition 6.5, HMSC $H$ in Fig. 10 is causally interferent and HMSC $H^{\prime}$ is not.

THEOREM 6.6. For a fixed set of processes $\mathbb{P}$, deciding causal non-interference of an HMSC $H$ with respect to a process $p \in \mathbb{P}$ is PSPACE-complete.

We prove this theorem in several steps. We first use the result of Proposition 3.6, i.e., the fact that black/white coloring of processes at the end of a sequence of concatenated MSCs can be done by remembering the status of processes after each MSC. This property holds for MSCs built along paths of HMSCs, and is used (in Proposition 6.7) to build HMSCs that recognize MSCs in $\mathcal{F}_{H}$ after which a fixed process is black (or similarly remains white). These HMSCs contain nodes of $H$, but remember for each node $n$ whether processes are black or white after an MSC built along a path ending in $n$. Then causal interference is reduced to an inclusion problem of finite automata that recognize sequences of actions along a process.

Proposition 6.7. Let $H$ be an HMSC, $p \in \mathbb{P}$, and $\Sigma=C \uplus V \uplus N$. Then, one can build:

- an HMSC $H^{B, p}$ that recognizes MSCs from $\mathcal{F}_{H}$ after which $p$ is a black process.

- an HMSC $H^{W, p}$ that recognizes MSCs from $\mathcal{F}_{H}$ after which $p$ is a white process.

of sizes in $O\left(|H| .2^{|\mathbb{P}|}\right)$.

Proof. (Sketch) The nodes of the HMSCs built in the proof memorize a node of the original HMSC, to which is added information on the color of each process: according to Proposition 3.6, this is the only information needed to remember the color of all processes in an MSC $M_{\rho}$ assembled along a path $\rho$ of $H$. The HMSC is furthermore equipped with accepting nodes that require $p$ to be black in $H^{B, p}$, and white in $H^{W, p}$.

We are now ready to prove theorem 6.6:

Proof. (of theorem 6.6) Following the construction of $H^{B, p}$ or $H^{W, p}$, we can define automata $\mathcal{A}_{p}^{B, p}$ and $\mathcal{A}_{p}^{W, p}$ that recognize the projections of $H^{B, p}$ or $H^{W, p}$ on process $p$. Let us denote by $\mathcal{O}^{B, p}(H)=\left\{\mathcal{O}^{p}(M) \mid M \in \mathcal{F}_{H} \wedge p\right.$ is black after $\left.M\right\}$ the observation function that returns the projection and by $\mathcal{O}^{W, p}(H)=\left\{\mathcal{O}^{p}(M) \mid M \in \mathcal{F}_{H} \wedge p\right.$ is white after $\left.M\right\}$. Clearly, we have $\mathcal{L}\left(\mathcal{A}_{p}^{B, p}\right)=\mathcal{O}^{p}\left(H^{B, p}\right)=\mathcal{O}^{B, p}(H)$ and $\mathcal{L}\left(\mathcal{A}_{p}^{W, p}\right)=\mathcal{O}^{p}\left(H^{W, p}\right)=\mathcal{O}^{W, p}(H)$, so $\mathcal{O}^{B, p}(H)$ and $\mathcal{O}^{W, p}(H)$ are recognized by finite automata.

Deciding causal interference of $H$ with respect to $p \in \mathbb{P}$ consists in deciding the inclusion prob-

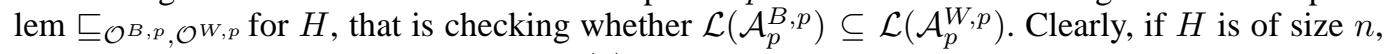
then $H^{B, p}$ and $H^{W, p}$ are of size in $O\left(n .2^{|\mathbb{P}|}\right)$, and so are $\mathcal{A}_{p}^{B, p}$ and $\mathcal{A}_{p}^{W, p}$. Then, checking inclusion of $\mathcal{L}\left(\mathcal{A}^{B, p}\right)$ into $\mathcal{L}\left(\mathcal{A}_{p}^{B, p}\right)$ is equivalent to checking $\mathcal{L}\left(\mathcal{A}_{p}^{B, p}\right) \cap \mathcal{L}\left(\overline{\mathcal{A}^{B, p}}\right)=\emptyset$. Emptiness of regular language is an NLOGSPACE problem, but the size of the automaton that recognizes the intersection is in $O\left(n .2^{|\mathbb{P}|} \cdot 2^{n .2^{|\mathbb{P}|}}\right)$, that is inclusion can be performed with space in $O\left(\log (n)+|\mathbb{P}|+n .2^{|\mathbb{P}|}\right)$. For a fixed set of processes, the space needed to check causal interferences is hence polynomial in the size of the input HMSC.

Like for local non-interference, the hardness result can be proved by polynomial encoding of a regular language inclusion problem. Given two regular languages $L_{1}, L_{2}$, one first designs two HMSCs $H_{1}, H_{2}$ with initial nodes $n_{0}^{1}, n_{0}^{2}$ such that $\mathcal{O}^{p}\left(H_{i}\right)=L_{i}$, for $i \in\{1,2\}$. Then, using again 
the construction illustrated in Fig. 7, we consider MSC $M_{c}^{\prime}$ that contains one confidential event on some fresh process $P_{c} \notin \mathbb{P}$, followed by messages from $P_{c}$ to all processes in $\mathbb{P}$, and at last, an HMSC $H$ that contains all transitions and accepting nodes of $H_{1}, H_{2}$, an initial node $n_{0}$ and an additional transition $t_{1}=\left(n_{0}, M_{c}^{\prime}, n_{0}^{1}\right)$. Any path of $H$ starting with transition $t_{1}$ generates an MSC in which $p$ is black, and whose projection on $p$ is in $L_{1}$. Other paths that do not start with $t_{1}$ generate MSCs from $\mathcal{F}_{H_{2}}$, and in particular MSCs in which $p$ is white and whose projection on $p$ is in $L_{2}$. Hence, $H$ is causally interferent with respect to $p$ if and only if $L_{1} \subseteq L_{2}$.

Causal interference can be checked in space in $O\left(\log (|H|)+|\mathbb{P}|+|H| \cdot 2^{|\mathbb{P}|}\right)$. This space complexity is polynomial in the size of the HMSC, and exponential in the number of processes, but HMSC specifications are usually defined for small sets of processes. Also remark that as soon as $V=\Sigma_{p}$, we can easily reuse the construction of $H^{W, p}$ to get a (word) automaton recognizing $\mathcal{O}_{\backslash C}^{V, \circ}(H)$.

\section{DECLASSIFICATION}

Non-interference considers confidential information as secrets that should remain undisclosed along all runs of a system. This point of view is too strict to be of practical interest: In many cases, confidentiality of a secret action has a limited duration and secrets can be downgraded. Consider the following example: a user wants to buy an item online, and pays by sending his credit card information. Everything from this transaction between the online shop and the buyer (even if encryption is used) should remain secret. Within this setting, all payment steps should be considered confidential, and flow from these actions to observable events should be prevented. However, if a buyer uses a one time credit card (i.e. a virtual credit card number generated on request that can be used only once for a transaction), then all information on the card is valueless as soon as the payment is completed. Hence, after completing the transaction, learning that a payment occurred is harmless and the sequence of interactions implementing a secured online payment need not be kept secret. This declassification possibility was first proposed as conditional interference by [Goguen and Meseguer 1982] and later defined in [Rushby 1992] as intransitive interference. Intransitive non interference (INI) can be formulated as follows: for any run of the system containing a confidential action that is not downgraded subsequently, there is a run with no classified action (all confidential actions are downgraded) which is equivalent from the observer's point of view.

Usually, INI is defined using a pruning function that removes from a run all confidential actions that are not declassified, and compares observations of pruned and normal runs (see [Gorrieri and Vernali 2011] for a definition of INI on transition systems). From now on, we assume that the alphabet $\Sigma=C \uplus V \uplus N$ contains a particular subset $D \subseteq V \uplus N$ of declassification events. Intuitively, declassification events downgrade all their confidential causal predecessors.

Definition 7.1. Let $M$ be an MSC. An event $e \in E_{M}$ is classified if it is a confidential event $(\alpha(e) \in C)$, it has an observable successor $v(\alpha(v) \in V)$ and it is not declassified before $v$, i.e., there exists no $d$ such that $e \leq d \leq v$ and $\alpha(d) \in D$. We denote by $C l a s(M)$ the set of classified events of $M$. The observation function $\mathcal{O}_{\backslash C, D}^{V}$ is defined by $\mathcal{O}_{\backslash C, D}^{V}(M)=\mathcal{O}^{V}(M \backslash$ Clas $(M))$. An MSC $M$ is intransitively non-interferent (INI) iff $\mathcal{O}_{\backslash C, D}^{V}(M)=\mathcal{O}^{V}(M)$.

We can characterize INI in a single MSC $M$ as a property depending on the causal order in $M$ and on the sets of confidential, declassification, and observable events.

PROPOSITION 7.2. An MSC $M$ is intransitively non-interferent w.r.t. an alphabet $\Sigma=C \uplus V \uplus$ $N$ and a set of declassification letters $D$ iff for every pair of events $c \leq v$ such that $\alpha(c) \in C$ and $\alpha(v) \in V$, we have $(\uparrow(c) \cap \downarrow(v)) \cap \alpha^{-1}(D) \neq \emptyset$.

This proposition means that a declassification must occur between every confidential event and a causally related visible event. We now define observation functions for HMSCs that consider declassification, and propose a definition of intransitive non interference for HMSCs. We define $\mathcal{O}_{I I, D}^{V}(H)=\left\{\mathcal{O}^{V}(M) \mid M\right.$ is not $\left.I N I\right\}$ and $\mathcal{O}_{I N I, D}^{V}(H)=\left\{\mathcal{O}^{V}(M) \mid M\right.$ is $\left.I N I\right\}$. We follow the 
definition of [Gorrieri and Vernali 2011] to define INI for HMSCs. An HMSC is INI if for every intransitively interferent (II for short) MSC $M$ in $\mathcal{F}_{H}$, there exists another MSC $M^{\prime}$ in $\mathcal{F}_{H}$ such that $M^{\prime}$ is INI and $\mathcal{O}^{V}\left(M^{\prime}\right)=\mathcal{O}^{V}(M)$.

Definition 7.3. An HMSC is intransitively non-interferent w.r.t. a declassification alphabet $D$ if $\mathcal{O}_{I N I, D}^{V}(H)=\mathcal{O}^{V}(H)$.

Obviously, $\mathcal{O}_{I N I, D}^{V}(H) \subseteq \mathcal{O}^{V}(H)$, so proving INI boils down to proving $\mathcal{O}^{V}(H) \subseteq \mathcal{O}_{I N I, D}^{V}(H)$. Note that all II MSCs are also interferent, and that checking non-interference amounts to checking INI with $D=\emptyset$. This remark extends to HMSCs: all intransitively interferent HMSCs are also causally interferent, and checking causal interference amount to checking INI with $D=\emptyset$. We then establish the following result:

THEOREM 7.4. INI for HMSCs is undecidable. For a fixed set of processes, if $V \subseteq \Sigma_{p}$, then INI is PSPACE-complete.

We prove the decidability part of this theorem in three steps detailed below. We first show that INI can be decided for a sequence of MSCs by remembering only the shape of causal chains originating from confidential events instead of the whole sequence. We then show that one can design an HMSC $H^{I I}$ that recognizes II MSCs of $\mathcal{F}_{H}$, and similarly an HMSC $H^{I N I}$ that recognizes INI MSCs of $\mathcal{F}_{H}$. An immediate consequence is that $\mathcal{O}_{I N I, D}^{V}(H)$ can be recognized by a finite automaton if $V \subseteq \Sigma_{p}$. A second consequence is that checking INI is PSPACE-complete. Let us first show that INI can be decided in a compositional way.

Proposition 7.5. Let $M_{1}, M_{2}$ be two MSCs. Then, $M_{1} \circ M_{2}$ is INI if and only if $M_{1}$ and $M_{2}$ are INI, and for each pair of events $c \in M_{1}, v \in M_{2}$ such that $\alpha(c) \in C, \alpha(v) \in V$, and $c \leq_{1 \circ 2} v$, there exists a process $q$, with

$-c \leq f$, where $f$ is the maximal event on process $q$ in $M_{1}$,

$-f^{\prime} \leq v$, where $f^{\prime}$ is the minimal event on $q$ in $M_{2}$,

and an event $d$ such that $\alpha(d) \in D$, and $c \leq d \leq f$ or $f^{\prime} \leq d \leq v$.

This proposition can be intuitively seen as a property of causal chains. Recall that a causal chain from $c$ to $v$ is a sequence of events $c \leq e_{1} \leq \ldots e_{n} \leq v$. We say that a chain from $c$ to $v$ is declassified if $\alpha\left(e_{i}\right) \in D$ for some $i \in 1$..n. Then an MSC is INI if for any pair $(c, v)$ of confidential/visible events such that $c \leq v$ there exists at least one declassified causal chain from $c$ to $v$. If so, the confidential event $c$ is guaranteed to be declassified by the occurrence of some declassifying action before the execution of $v$ occurs.

A causal chain from $c$ to $v$ in $M_{1} \circ M_{2}$ can be decomposed into a chain from $c$ to the maximal event $f$ on a process $q$ in $M_{1}$, a causal ordering from $f$ to a minimal event $f^{\prime}$ located on process $q$ in $M_{2}$ coming from the sequential composition of $M_{1}$ and $M_{2}$, and then a causal chain from the minimal event $f^{\prime}$ on $q$ to $v$. However, one does not need to know precisely the contents of $M_{1}$ to decide whether $M_{1} \circ M_{2}$ is INI. It suffices to remember for each process $p$ the confidential events of $M_{1}$ that are not yet declassified and are predecessors of the maximal event executed by process $p$ in $M_{1}$.

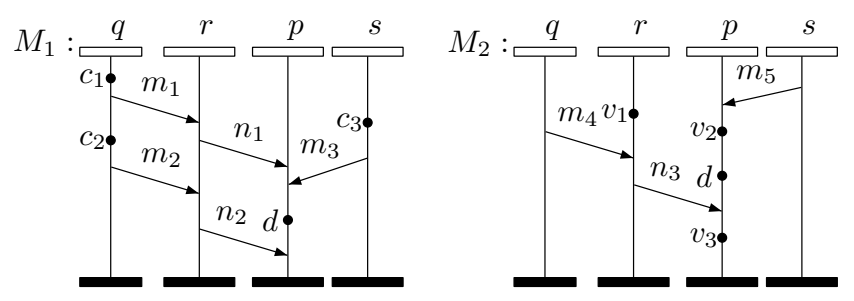

Fig. 11. An example of non INI sequence of MSCs 
On the example depicted in Fig. 11, MSC $M_{1}$ (left) contains three confidential actions $c_{1}, c_{2}, c_{3}$, and a declassification operation $d$. On the right, MSC $M_{2}$ contains three visible actions $v_{1}, v_{2}, v_{3}$, and a declassification operation $d$. All other events belong to $\alpha^{-1}(N)$. Both MSCs are INI, since no observation depends on a confidential action in $M_{1}$ or in $M_{2}$. However, in the concatenation $M_{1} \circ M_{2}$, execution of $v_{1}$ or $v_{2}$ reveals the occurrence of $c_{2}$. Also note that $c_{1}$ is declassified by the first occurrence of $d$ in $M_{1}$. This example is particularly interesting, as it shows that in order to abstract an arbitrarily long execution, it is not sufficient to remember a boolean value indicating whether there exists a not yet declassified action on a process, as two confidential events on the same process can be declassified by different causal chains. Indeed, some confidential actions could be declassified for a process while some others could not, even when located on the same process.

We can characterize II MSCs in a set $\mathcal{F}_{H}$ by remembering at each step of a sequence of MSCs only a finite sets of shapes of causal chains. In order to define these shapes, let $M$ be an MSC, let $c$ be a confidential event in $M$. We define a function $c l(c, M): \mathbb{P} \longrightarrow\{\perp,+, \top\}$ such that $c l(c, M)(p)=\perp$ if there exists no causal chain from $c$ to an event located on $p, c l(c, M)(p)=+$ if there exists a causal chain from $c$ to a maximal event $f$ located on $p$, and $(\uparrow c \cap \downarrow f) \cap \alpha^{-1}(D)=\emptyset$, and $\operatorname{cl}(c, M)(p)=\top$ otherwise. This function classifies processes according to the existence and classification degree (declassified or not) of causal chains between the confidential event $c$ and the last event seen on each process. For a set $\mathbb{P}$ of processes, any such map $c l(c, M)$ can have at most $3^{|\mathbb{P}|}$ distinct values. Let $\mathcal{C l}=\{\perp,+, \top\}^{\mathbb{P}}$ denote the set of all maps. By proposition $7.5, M_{1} \circ M_{2}$ is not INI if $M_{1}$ or $M_{2}$ is not INI, or if there exists $c \in M_{1}$ and $v \in M_{2}$ such that:

- there exists a process $p$ such that $c l\left(c, M_{1}\right)(p)=+$, and an event $f$ located on $p$ in $M_{2}$, such that no causal chain from $f$ to $v$ is declassified.

- for every process $q$ such that $c l\left(c, M_{1}\right)(q)=\top$ there exists no event $f \leq v$ located on $q$ in $M_{2}$, and $v$ is not located on $q$.

One can furthermore compute $\operatorname{cl}\left(c, M_{1} \circ M_{2} \circ \cdots \circ M_{k}\right)(p)$ incrementally with finite memory: $\operatorname{cl}\left(\mathbf{c}, \mathbf{M}_{1} \circ \mathbf{M}_{\mathbf{2}}\right)(\mathbf{p})=\perp$ if $c l\left(c, M_{1}\right)(p)=\perp$, and if there exists no pair of events $e \leq f$ in $M_{2}$ with $f$ is located of $p$, and $c l\left(c, M_{1}\right)(\phi(e)) \neq \perp$.

$\operatorname{cl}\left(\mathbf{c}, \mathbf{M}_{\mathbf{1}} \circ \mathbf{M}_{\mathbf{2}}\right)(\mathbf{p})=+$ if $\operatorname{cl}\left(c, M_{1}\right)(p) \in\{\perp,+\}$, there exists a process $q$ such that $c l\left(c, M_{1}\right)(q)=+$, and a pair of events $e \leq f$ in $M_{2}$ such that $e$ is minimal on $q$, $f$ is maximal on process $p$, and furthermore, no causal chain from $e$ to $f$ is declassified, and for every process $q^{\prime} \neq q$, if $c l\left(c, M_{1}\right)\left(q^{\prime}\right)=+$, then no declassified causal chain from an event on $q^{\prime}$ to $f$ exists in $M_{2}$, if $c l\left(c, M_{1}\right)\left(q^{\prime}\right)=\top$ then no causal chain from an event on $q^{\prime}$ to $f$ exists in $M_{2}$.

$\operatorname{cl}\left(\mathbf{c}, \mathbf{M}_{\mathbf{1}} \circ \mathbf{M}_{\mathbf{2}}\right)(\mathbf{p})=\top$ if $c l\left(c, M_{1}\right)(p)=\top$, or

- there exist a process $q$ such that $c l\left(c, M_{1}\right)(q)=+$ and a declassified chain from an event $e$ located on process $q$ to an event $f$ located on process $p$, or

- there exist a process $q$ such that $c l\left(c, M_{1}\right)(q)=\top$, and a causal chain from an event $e$ located on process $q$ to an event $f$ located on process $p$.

Last, $\operatorname{cl}\left(c, M_{1} \circ M_{2}\right)(p)=\perp$ if $\operatorname{cl}\left(c, M_{1}\right)(p)=\perp$ and $M_{2}$ does not contain a pair of events $e \leq f$ such that $e$ is located on $q$ with $\operatorname{cl}\left(c, M_{1}\right)(q) \neq \perp$, and $f$ is located on $p$.

Now, if $M_{1}$ contains two confidential events $c_{1}, c_{2}$ such that $\operatorname{cl}\left(c_{1}, M_{1}\right)=\operatorname{cl}\left(c_{2}, M_{1}\right)$, then $\operatorname{cl}\left(c_{1}, M_{1} \circ M_{2}\right)=\operatorname{cl}\left(c_{2}, M_{1} \circ M_{2}\right)$. It means that to detect interferences, one does not have to remember events, but only the shape of causal relations (existing, declassified or not) from confidential events to their successors on each process. Furthermore, at most $3^{|\mathbb{P}|}$ distinct shapes can appear in an MSC, so one can check INI along arbitrarily long sequences of MSCs with finite memory.

Proposition 7.6. Let $H$ be an HMSC, with labeling alphabet $\Sigma$ and set $D$ of declassification letters. Then, one can build an HMSC $H^{I I}$ generating all II MSCs in $\mathcal{F}_{H}$ and an HMSC $H^{I N I}$ generating all INI MSCs in $\mathcal{F}_{H}$, with sizes at most $2 .|H| .2^{3^{|\mathbb{P}|}}$ 
PRoOF. (Sketch) We build HMSC $H^{I I}$ as follows: a state $(n, b, X)$ of $H^{I I}$ memorizes a node $n$ of $H$, a boolean $b$ indicating whether an interference has been detected, and a set $X=\left\{c l_{1}, \ldots c l_{\ell}\right) \subseteq$ $\mathcal{C l}$, where each $c l_{i}$ is a function from $\mathbb{P}$ to $\{\perp,+, \top\}$ that memorizes the shape of causal chains from a confidential event to maximal events on processes. $H^{I I}$ follows transitions of $H$, and updates $c l_{i}$ 's. For each new confidential event $c$ occurring in a transition labeled by an MSC $M$, a new function $c l(c, M)$ is added to memorized shapes in $X$. As soon as an interference is detected, $b$ is set to true. Accepting states of $H^{I I}$ are of the form $(n, b, X)$ where $n$ is accepting in $H$, and $b$ is true. $H^{I N I}$ is built similarly, but with accepting states of the form $(n, b, X)$ with $n$ accepting in $H$ and $b$ false.

We are now ready to prove Theorem 7.4:

PROOF. (of Theorem 7.4) Undecidability is easily obtained from undecidability of causal interference, and by setting $D=\emptyset$. Let us now consider the decidability part, with $V \subseteq \Sigma_{p}$. Following the proof of proposition 7.6, one can build an automaton $\mathcal{A}_{p}\left(H^{I N I}\right)$ of size at most $2 .|H| .2^{\mid{ }^{|\mathbb{P}|}}$ that recognizes $\mathcal{O}^{V}\left(H^{I N I}\right)$. One can easily prove that when $V \subseteq \Sigma_{p}$, we have $\mathcal{O}^{V}\left(H^{I N I}\right)=\mathcal{O}_{I N I, D}^{V}(H)$, and hence $\mathcal{L}\left(\mathcal{A}_{p}\left(H^{I N I}\right)\right)=\mathcal{O}_{I N I, D}^{V}(H)$, i.e. $\mathcal{O}_{I N I, D}^{V}(H)$ is recognized by a finite automaton.

From proposition 6.2, we can build an automaton $\mathcal{A}_{p}(H)$ of size in $O(k .|H|)$, where $k$ is the maximal number of events in an MSC of $H$, that recognizes $\mathcal{O}^{V}(H)$. Then it is sufficient to check whether $\mathcal{L}\left(\mathcal{A}_{p}(H)\right) \subseteq \mathcal{L}\left(\mathcal{A}_{p}\left(H^{I N I}\right)\right)$ to decide if $H$ is intransitively interferent, which is again an inclusion problem that can be checked in space in $O\left(2 .|H| \cdot 2^{3^{|\mathbb{P}|}}\right)$. Recalling that $\mathbb{P}$ is fixed, the space needed to check intransitive interference is hence linear w.r.t. the size of the original HMSC. Hardness is proved by showing a polynomial reduction from a language inclusion problem to an INI problem with $D=\emptyset$.

The declassification setting can be refined to consider selective declassification. Following the definition of [Best and Darondeau 2012], in addition to the declassification alphabet $D$, we define a map $h: D \rightarrow 2^{C}$, where $h\left(\alpha_{d}\right)$ defines the labels of confidential events that an action with label $\alpha_{d}$ declassifies. Definition 7.1 easily adapts to this setting, simply by requiring that a causal chain from a confidential event $c$ to a visible event $v$ is declassified by an event $d$ such that $\alpha(c) \in h(\alpha(d))$. We then say that an event $c$ is classified if it is a confidential event $(\alpha(c) \in C)$, it has an observable successor $v$, and it is not declassified by one of the actions that can declassify it, that is, $\alpha(c) \notin$ $h(\alpha(\uparrow(c) \cap \downarrow(v)) \cap D)$. INI with selective declassification (INISD) adapts the definitions of INI to consider declassification without changing observations. Like for standard declassification, we can build an HMSC that recognizes INISD MSCs of $\mathcal{F}_{H}$. The only change w.r.t. INI is that one has to remember in the HMSC construction the label of confidential events from which chains originate, yielding automata of sizes in $2 .|H| .2^{|C| .3^{|\mathbb{P}|}}$. If $V \subseteq \Sigma_{p}$, then $\mathcal{O}_{I I, D}^{V}$ and $\mathcal{O}_{I N I, D}^{V}$ are recognized by finite automata. We hence have:

COROLLARY 7.7. INISD is undecidable for HMSCs. For a fixed set of processes, it is PSPACEcomplete when $V \subseteq \Sigma_{p}$.

\section{RELATED WORK AND CONCLUSION}

Related work. Non-interference was seldom studied for scenario formalisms. A former work considers non-interference for Triggered Message Sequence Charts [Ray et al. 2004]. The interference property is defined in terms of comparison of ready sets (sets of actions that are fireable after a given sequence of actions $w$ ). However, this work mainly considers finite scenarios, and does not address decidability and complexity issues.

A first work considering non-interference for true concurrency models appears in [Busi and Gorrieri 2009]. The authors consider interference for elementary nets (i.e., nets where firing rules allow places to contain at most one token). They characterize causal places, where firing a highlevel transition causally precedes the firing of a low-level one and conflict places, where firing a high-level transition inhibits the firing of a low-level one. Reachability of causal or conflict places 
is shown equivalent to BNDC (Bisimulation-based NDC, the variant of Non-Interference using bisimulation instead of language equality). In [Gorrieri and Vernali 2011], the notion of intransitive non-interference from [Rushby 1992] is revisited for transition systems, and non-interference with downgraders is considered for elementary nets. A structural characterization is given in terms of reachable causal and conflict places. As in [Busi and Gorrieri 2009], causal and conflict places are characterized in terms of possible fireable sequences of transitions, hence considering the interleaving semantics of the net.

Darondeau et al. [Best et al. 2010] study (B)NDC and INI for unbounded labeled Petri nets, and extend their results to selective declassification in [Best and Darondeau 2012]. They obtain decidability of these properties for injectively labeled nets by a very clever exploitation of specific decidability results for language inclusion, which is undecidable for general Petri nets languages. The characterization relies on sequences of transitions, and not on causal properties of nets.

A contrario, Baldan et al [Baldan and Carraro 2014] emphasize the fact that characterizing BNDC in terms of structural conditions expressing causality or conflict between high and low-level transitions, is a way to provide efficient algorithms to check interference. They propose a definition of complete unfolding w.r.t. non-interference, and reduce BNDC for safe nets to checking that a complete unfolding is weak-conflict and weak-causal place free. Weak causal places characterize dependencies and conflicts between high and low transitions. Their results show that interference can be detected in concurrent models without relying on interleaving semantics. They only hold for safe nets, i.e., for finite state systems.

Conclusion. We proposed a partial order framework for information flow properties analysis, that relies on comparisons of sets of partial orders describing observations of system executions. We proved that inclusion of observed orders and non-interference are undecidable in general. To alleviate this problem, we proposed partial order automata, as a model to recognize observations of executions. We then identified subclasses of partial order automata for which language inclusion is decidable. Locally synchronized HMSCs falls into this category, hence non interference is decidable in this subclass. A different approach to obtain decidability in this partial order framework is to restrict the kind of observation functions that can be used. This is a sensible approach, as it amounts to restricting the power of attackers. When visible events are observed by a single process of the system, most of observation functions applied to HMSCs define regular languages. As a consequence, several notions of local non-interference and their extensions with declassification, are decidable. We showed that local versions of non-interference are PSPACE-complete problems, and give decision procedures that never compute the interleaving semantics of the original HMSC.

So far, partial order automata are mainly used as an intermediate technicality to prove decidability of non-interference for locally-synchronized HMSCs when several processes can be observed in a system. However, this model is more general than HMSCs. A possible refinement of the landscape is to consider decidability of interference for partial order automata that generate sets of orders with non-regular linearization languages. We conjecture that decidability of inclusion can be generalized to some subclasses of non-regular partial order automata, some classes of graph grammars, or more generally to subclasses of models with bounded-split width [Aiswarya et al. 2014].

Another line of research is to consider stronger information flow properties in HMSCs. We have shown that local interference is weaker than causal interference, and that declassification allows finer definitions of information leakage. To overcome weakness of language-based information flow characterizations, the notion of NDC (Non-Deducibility on Composition) was proposed to detect when confidential actions cause observable effects. Informally, NDC says that a system $S$ composed with any machine $R$ (that enables/forbids confidential events) is observationally equivalent to $S$. At first glance, causal non-interference appears weaker than a causal form of NDC: it compares the observations of an HMSC with the observations that are still possible without confidential events. With respect to this definition, it is the comparison of the behavior of a specification controlled by one machine that prevents all confidential events. In contrast, NDC compares a behavior of a specification with the behaviors of the same specification controlled by every possible high-level mechanism, which can prevent some confidential events to occur. Hence, specifications that are not 
causally interferent may nevertheless disclose information when controlled by other machines. So defining a causal form of NDC for HMSCs along the lines sketched in [Baldan and Carraro 2014] is an appealing task.

Finally, we could consider security issues when an attacker can interact with the system in order to gain information (active interference), or when he can get information on the current configuration of the system (state-based interference). Extending definitions of information flows in HMSCs to quantify the amount of information disclosure by mean of measures (e.g., probability measures, average number of bits leaked per action,...) is also a challenging perspective.

\section{REFERENCES}

C. Aiswarya, P. Gastin, and K. Narayan Kumar. 2014. Verifying Communicating Multi-pushdown Systems via Split-Width. In Proc. of ATVA 2014 (LNCS), Vol. 8837. 1-17.

R. Alur and M. Yannakakis. 1999. Model Checking of Message Sequence Charts. In Proc. of 10th Int. Conf. on Concurrency Theory, CONCUR '99 (LNCS), Vol. 1664. 114-129.

P. Baldan and A. Carraro. 2014. Non-interference by Unfolding. In 35th Int. Conf. on Application and Theory of Petri Nets and Concurrency, PETRI NETS (LNCS), Vol. 8489. 190-209.

B. Bérard, L. Hélouët, and J. Mullins. 2015. Non-interference in Partial Order Models. In Proc. of 15th Int. Conf. on Application of Concurrency to System Design, ACSD 2015. IEEE Computer Society, 80-89.

E. Best and P. Darondeau. 2012. Deciding Selective Declassification of Petri Nets. In Proc. of 1st Int. Cong. on Principles of Security and Trust, POST 2012 (LNCS), Vol. 7215. 290-308.

E. Best, P. Darondeau, and R. Gorrieri. 2010. On the Decidability of Non Interference over Unbounded Petri Nets. In Proc. of SecCo (EPTCS), Vol. 51. 16-33.

B. Bérard and J. Mullins. 2014. Verification of Information Flow Properties under Rational Observation. In Proc. of AVOCS 2014, ECEASST 70.

N. Busi and R. Gorrieri. 2009. Structural non-interference in elementary and trace nets. Mathematical Structures in Computer Science 19, 6 (2009), 1065-1090.

B. Caillaud, P. Darondeau, L. Hélouët, and G. Lesventes. 2000. HMSCs en tant que spécifications partielles et leurs complétions dans les réseaux de Petri. RR-3970. INRIA.

D. D'Souza, R. Holla, K.R. Raghavendra, and B. Sprick. 2011. Model-checking trace-based information flow properties. Journal of Computer Security 19, 1 (2011), 101-138.

R. Focardi and R. Gorrieri. 2001. Classification of Security Properties (Part I: Information Flow). In Foundations of Security Analysis and Design (LNCS), Vol. 2171. Springer-Vale, 331-396.

B. Genest, L. Hélouët, and A. Muscholl. 2003. High-Level Message Sequence Charts and Projections. In Proc. of CONCUR'03 (LNCS), Vol. 2761. 308-322.

J.A. Goguen and J. Meseguer. 1982. Security policies and security Models. In Proc. of IEEE Symposium on Security and Privacy. 11-20.

R. Gorrieri and M. Vernali. 2011. On Intransitive Non-interference in Some Models of Concurrency. In FOSAD VI Tutorial Lectures (LNCS), Vol. 6858. 125-151.

L. Hélouët, H. Marchand, B. Genest, and T. Gazagnaire. 2014. Diagnosis from scenarios. Discrete Event Dynamic Systems 24, 4 (2014), 353-415.

ITU-T. 2011. Z.120 : Message Sequence Charts (MSC). Technical Report. International Telecommunication Union.

H. Mantel. 2000. Possibilistic Definitions of Security - An Assembly Kit. In Proc. of the 13th IEEE Computer Security Foundations Workshop, (CSFW'00). 185-199.

H. Mantel. 2001. Information Flow Control and Applications - Bridging a Gap. In Proc. of FME 2001 (LNCS), Vol. 2021. 153-172.

F. Mattern. 1988. Time and global states of distributed systems. in Proc. Int. Workshop on Parallel and Distributed Algorithms, Bonas, France, North Holland (1988), 215-226.

A. Muscholl and D. Peled. 1999. Message Sequence Graphs and Decision Problems on Mazurkiewicz Traces. In Proc. of 24th Int. Conf. on Mathematical Foundations of Computer Science, MFCS (LNCS), M. Kutylowski, L. Pacholski, and T. Wierzbicki (Eds.), Vol. 1672. 81-91.

A. Muscholl and D. Peled. 2000. Analyzing Message Sequence Charts. In Proc. of 2nd Workshop on SDL and MSC, SAM 2000. 3-17.

A. Ray, B. Sengupta, and R. Cleaveland. 2004. Secure Requirements Elicitation Through Triggered Message Sequence Charts. In Proc. of ICDCIT 2004 (LNCS), Vol. 3347. 273-282.

J. Rushby. 1992. Noninterference, Transitivity, and Channel-control Security Policies. Technical Report CSL-92-02. SRI International. 